\title{
Unsteady Flow and Aerodynamic Effect of a Dynamic Trailing-Edge Flap in Flapping Flight
}

\author{
A Thesis \\ Presented to \\ the faculty of the School of Engineering and Applied Science \\ University of Virginia
}

In Partial Fulfillment

of the requirements for the Degree

Master of Science in Mechanical and Aerospace Engineering

By

Chengyu Li

May 2014 


\section{APPROVAL SHEET}

The thesis is submitted in partial fulfillment of the

requirements for the degree of Master of Science

Chengyu Li, Author

This thesis has been read and approved by the examining Committee:

\begin{tabular}{c}
\hline Haibo Dong, Advisor \\
\hline Christopher P. Goyne, Committee Chair \\
\hline Gavin Garner, Committee Member
\end{tabular}

Gavin Garner, Committee Member

Accepted for the School of Engineering and Applied Science:

James H. Aylor, Dean

School of Engineering and Applied Science

May 2014 


\begin{abstract}
Flapping-wing mechanism offers unique aerodynamic advantages over conventional flight methods for design of micro air vehicles (MAVs). The inherently unsteady nature of the flapping motion is responsible for the primary force production, and also differentiates flapping wing fliers from conventional fixed and rotary wing configurations. The unsteady aerodynamic phenomena are not only sensitive to variations in the wing motion but also for the dynamic deformed wing surface. Yet, it is not well understood how the unsteady aerodynamic phenomena and dynamic wing morphing interact to modulate flow and to determine overall aerodynamic performance.

In current work, the deformable wing is simplified as a rigid plate hinged with a controllable trailing-edge flap. The leading-edge portion of the plate is driven by a prescribed hovering motion moving in a horizontal stroke plane. The deflection of trailing-edge flap follows a sinusoidal function with respect to the leading-edge for mimicking time-varying camber deformation. Key parameters for determining deformed plate kinematics, such as stroke-to-chord ratio, trailing-edge flap deflection amplitude, and phase shift of trailing-edge deflection, are studied to explore their effects on aerodynamic performance and flow modulation. An in-house immersed boundary method based Direct Numerical Simulation (DNS) solver is used to simulate the unsteady flow. Results from current parametric studies will be used to analyze unsteady force productions due to dynamic trailing-edge flap in flapping flight.
\end{abstract}




\section{Acknowledgements}

I would like to express my heartfelt gratitude and sincere thanks to my research advisor Dr. Haibo Dong, Associate Professor of Mechanical Engineering, Department of Mechanical and Aerospace Engineering at University of Virginia. Without his guidance, constant supervision, and valuable suggestions throughout the entire period of the work, it would have been impossible to complete the research work. I am thankful to my parents for all their support throughout my life. I am also greatly thankful to Mr. Yan Ren, Ms. Samane Zeyghami, and all other Flow Simulation Research Group (FSRG) members for their assistance, generosity and advice throughout this study.

I am very grateful to the faculty and staff of the Department of Mechanical and Aerospace Engineering at University of Virginia for providing me with the opportunity to pursue higher studies. 


\section{Table of Contents}

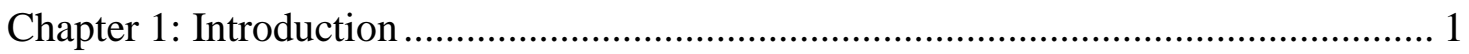

1.1 Unsteady Aerodynamics of Flapping Flight ..................................................... 2

1.2 Dynamic Deformed Wing in Flapping Flight .................................................. 3

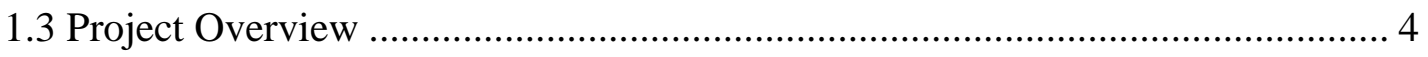

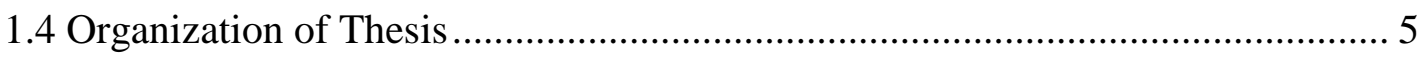

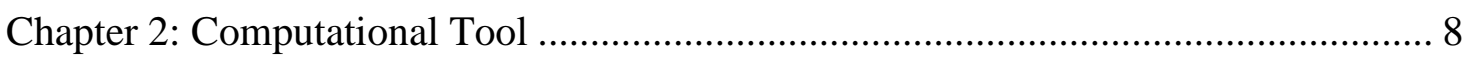

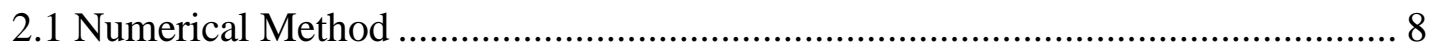

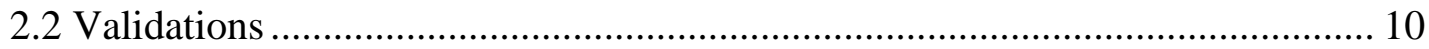

Chapter 3: Parametric Study of Two-Dimensional Hovering Plate with Trailing Edge

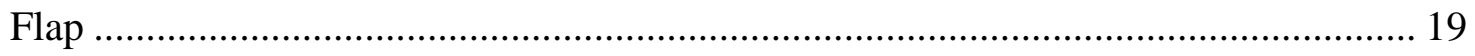

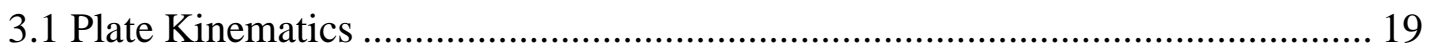

3.2 Normalization and Choice of Parameters ………............................................... 20

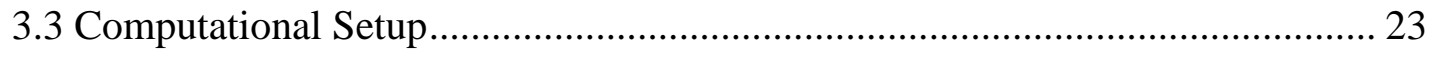

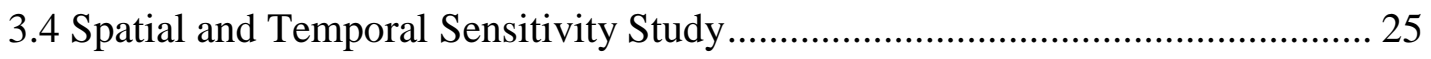

3.5 General Performance Comparison ................................................................ 27

3.6 Stroke Amplitude Effects................................................................................. 30

3.7 Effects of Trailing-Edge Flap Deflection Amplitude and Phase Shift .............. 36

3.8 Vortex Modulation Resulting from Trailing-Edge Flap ................................... 42

Chapter 4: Three-Dimensional Hovering Motion with Trailing-Edge Flap ................. 48 
4.1 Plate Geometry and Kinematics.............................................................. 48

4.2 Normalization and Choice of Parameters ..................................................... 50

4.3 Case Setup and Grid Independent Study ...................................................... 52

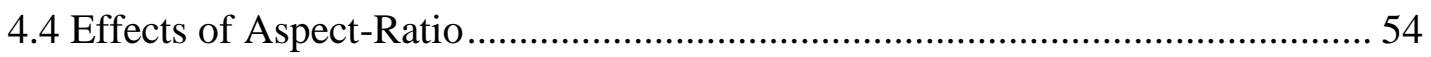

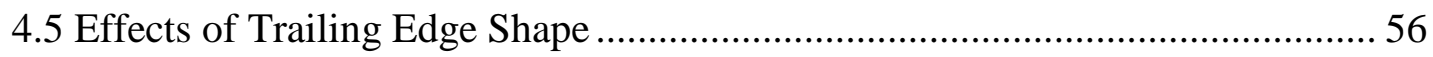

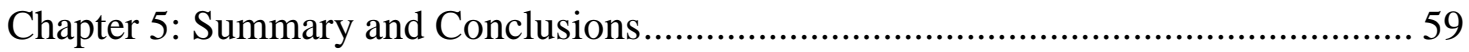

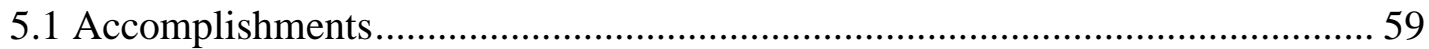

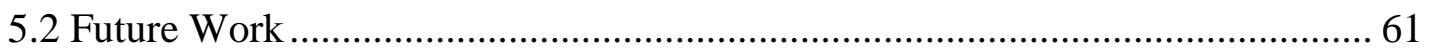

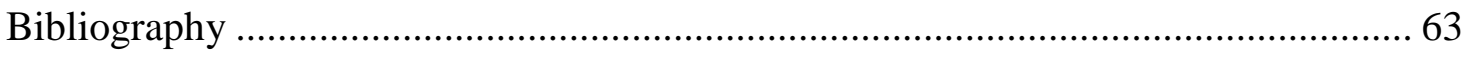




\section{Lift of Figures}

Figure 1: Variation of aerodynamic efficiency with Reynolds number. The aerodynamic efficiency of the smooth airfoil drops rapidly when Reynolds number is less than 100,000 (From Mcmasters, J. H. and Henderson, 1980[1])

Figure 2: (A) Hummingbird hovering flight, picture courtesy of J. M. Hughes; (B) Flying bat, picture courtesy of Duncan PJ; (C) Dragonfly in free flight showing large scale wing deformation, picture courtesy of H. Alberts; (D) Veinous wing structure of dragonfly, picture courtesy of F. L. Mitchell. ..................................................................... 7

Figure 3: Time history of lift and drag coefficients for rectangular plan-form at $\operatorname{Re}=300$, and $\alpha=30^{\circ}$. (A) Comparison of Lift coefficient; (B) Comparison of Drag coefficient. 12 Figure 4: Wake vortices behind a rectangular wing of $\mathrm{AR}=2$ at $\alpha=30^{\circ}$ and $\mathrm{Re}=300$. Shown are the iso-surface of $\mathrm{Q}=3$. (A) from [16]; (B) our simulation results. 13

Figure 5: Rectangular plate $(A R=2)$ translation configuration $(\mathrm{A})$ and velocity profile in time (B). 14

Figure 6: Time history of lift and drag coefficients for rectangular plan-form $(A R=2)$ in translational motion at $\operatorname{Re}=800$, and $\alpha=35^{\circ}, 45^{\circ}$ and $55^{\circ}$. (A) Comparison of Lift coefficient; (B) Comparison of Drag coefficient. 15

Figure 7: Rectangular plate $(A R=2)$ rotational configuration $(\mathrm{A})$ and angular velocity profile in time (B). 16

Figure 8: Time history of lift and drag coefficients for rectangular plan-form $(A R=2)$ in rotational motion at $\operatorname{Re}=500$, and $\alpha=45^{\circ}$. (A) Comparison of Lift coefficient; (B) Comparison of Drag coefficient. 18 
Figure 9: (A) Rigid plate hinged with a $25 \%$ chord length trailing-edge flap, $H$ is the lending-edge, $H_{L}$ denotes the length of leading-edge $\left(H H^{\prime}\right)$ which is $75 \%$ of total chord length; (B) Flapping plate configuration with $A_{0}=3.0, \beta_{T}=60^{\circ}$ and $\varphi=60^{\circ}$. 20

Figure 10: Plate flapping configuration without trailing-edge flap (TEF) (A), with symmetrical $\left(\varphi=0^{\circ}\right) \mathrm{TEF}$ rotation (B), with delayed $\left(0^{\circ}<\varphi<180^{\circ}\right)$ TEF rotation $(\mathrm{C})$ and with advanced $\left(-180^{\circ}<\varphi<0^{\circ}\right)$ TEF rotation. The red and blue color chords indicate the down-stroke and up-stroke, respectively.

Figure 11: (A) Flapping plate configuration immersed in the two-dimensional nonuniform Cartesian grid; (B) Boundary conditions for the simulation. 25

Figure 12: Spatial and Temporal sensitivity analyses for a rigid flapping plate at $A_{0} / c=2.0$. Grid sizes are: Coarse, $0.15 \times 10^{6}$ cells; Medium, $0.29 \times 10^{6}$ cells; and Fine, $0.61 \times 10^{6}$ cells. Lift coefficient $(A, C)$ and drag coefficient $(B, D)$ time history for the $10^{\text {th }}$ flapping stroke.

Figure 13: Comparison of idealized normal hovering for rigid plate (A) and hinged plate (B). (i) Wing motion; (ii) instantaneous vertical ( $\mathrm{CV}$ ) and horizontal ( $\mathrm{CH}$ ) force coefficients; (iii) snapshots of vorticity (red, counterclockwise rotation; blue, clockwise rotation) during the tenth period, and (iv) time-averaged velocity field 29

Figure 14: Stroke amplitude effects on lift coefficient (A) and drag coefficient (B) for rigid flat plates.

Figure 15: Cycle-averaged drag coefficient (A), lift coefficient (B), and aerodynamic power coefficient (C) plotted against the trailing-edge deflection amplitude $\left(\beta_{T}\right)$ for different flapping amplitude value $\left(A_{0} / c\right)$. In which, $\beta_{T}$ ranging from $10^{\circ}$ to $80^{\circ}$, and 
$\beta_{T}=0^{\circ}$ shows the performance of the rigid flat plate. In all above cases, the phase shaft $(\varphi)$ is zero and the Reynolds number was fixed at 100.

Figure 16: (A) Cycle-averaged lift-to-drag ratio; (B) Aerodynamic efficiency (lift-topower). In which, $\beta_{T}$ ranging from $10^{\circ}$ to $80^{\circ}$, and $\beta_{T}=0^{\circ}$ shows the performance of the rigid flat plate. In all above case the Reynolds number were fixed at 100. 35

Figure 17: Sample instantaneous lift $(A, C)$ and drag (B, D) coefficient for various combinations of trailing-edge deflection amplitude $\left(\beta_{T}\right)$ and phase shift $(\varphi)$. In each case, the flapping stroke amplitude is fixed at $A_{0} / c=3$, and Reynolds number is $100 \ldots 37$

Figure 18: Parameter maps of cycle-averaged lift coefficient (A), drag coefficient (B), and aerodynamic power coefficient $(\mathrm{C})$ as function $\mathrm{s}$ of trailing-edge deflection amplitude and phase shift

Figure 19: Parameter maps of cycle-averaged lift-to-drag ratio (A), and lift-to-power ratio (B) as function s of trailing-edge deflection amplitude and phase shift. 40

Figure 20: Instantaneous lift (A) and drag (B) coefficient for flapping plate without trailing-edge flap (Rigid), owning optimal cycle-averaged lift $\left(\beta_{T}=50^{\circ}, \varphi=+60^{\circ}\right)$, and owning optimal cycle-averaged aerodynamic efficiency $\left(\beta_{T}=10^{\circ}, \varphi=-80^{\circ}\right)$.each case, the flapping stroke amplitude is fixed at $A_{0} / c=3$, and Reynolds number is 100 . 41

Figure 21: A series of instantaneous vortex field in a flapping cycle for rigid plate (first and third columns) and plate with trialing-edge flap (second and fourth columns). The red and blue color indicates clockwise and anticlockwise, respectively. 43

Figure 22: Comparison of leading-edge vortex (LEV) circulation between rigid plate without trailing-edge flap (TEF) and with TEF dynamic motion $\left(\beta_{T}=50^{\circ}, \varphi=60^{\circ}\right)$. The 
LEV circulation is nondimensionalized using reference velocity $(U)$ and chord length (c). The gray shades indicate the down-stroke periods. The positive and negative values correspond to the strength of LEV during down-stroke and up-stroke, respectively....... 45

Figure 23: Pressure contour comparison between rigid plate (A) and plate hinged with trailing-edge flap (B). The pressure is normalized by using reference velocity. 46

Figure 24: Contour of mean velocity (in vertical direction) corresponding to plate flapping configurations. (A) Rigid plate without trailing-edge flap; (B) Plate with trailingedge flap dynamic motion at $\beta_{T}=50^{\circ}, \varphi=60^{\circ}$

Figure 25: Three-dimensional flapping plate kinematics. (A): Plate geometry and definition of kinematics parameters; (B): Flapping plate configuration during downstroke (red) and up-stroke (blue).

Figure 26: Three types of trailing-edge shape. (A) Rectangular shape (TS); (B) Left triangle shape (LTS); (C) Right triangle shape (RTS). 50

Figure 27: (A) Three-dimensional flapping plate configuration immersed in the twodimensional non-uniform Cartesian grid; (B) Boundary conditions for the simulation... 52 Figure 28: Comparison of instantaneous lift coefficient (A) and drag coefficient (B) for different aspect-ratio ( $A R=1,2,4)$ during the third and fourth flapping cycle. 55

Figure 29: Evolution of the surface pressure distribution on the suction side of the plate with different aspect-ratio. The pressure is normalized by the reference velocity. 56

Figure 30: Comparison of instantaneous lift coefficient (A) and drag coefficient (B) for different trailing-edge shape (Rigid: without trailing-edge flap; RS: rectangular trailingedge shape; LTS: left triangular trailing-edge shape: RTS: right triangular trailing-edge shape) during the third and fourth flapping cycle. 57 
Figure 31: Comparison of surface pressure distribution on the suction side of the plate (A) and iso-surface vortex structure (B) for different trailing-edge shape at the middle of

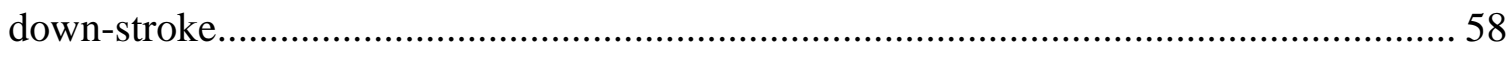




\section{Lift of Tables}

Table 1: Summary of lift and drag coefficient for flat flapping plates with different stroke amplitude...

Table 2: Comparative study of cases with and without TEF

Table 3: Comparison of mean lift coefficient $\left(\overline{C_{L}}\right)$, drag coefficient $\left(\overline{C_{D}}\right)$, aerodynamic power coefficient $\left(\overline{C_{P}}\right)$, lift-to-drag ratio $\overline{C_{L}} / \overline{C_{D}}$, and lift-to-power ratio $\overline{C_{L}} / \overline{C_{P}}$ for

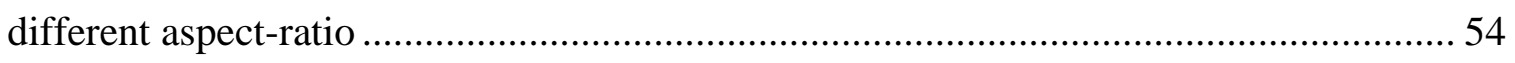

Table 4: Comparison of mean lift coefficient $\left(\overline{C_{L}}\right)$, drag coefficient $\left(\overline{C_{D}}\right)$, aerodynamic power coefficient $\left(\overline{C_{P}}\right)$, lift-to-drag ratio $\overline{C_{L}} / \overline{C_{D}}$, and lift-to-power ratio $\overline{C_{L}} / \overline{C_{P}}$ for different trailing-edge flap (TEF) shape. ..... 58 


\section{Nomenclature}

\begin{tabular}{|c|c|}
\hline Symbol & Description \\
\hline $2-\mathrm{D}$ & Two dimensional \\
\hline $3-\mathrm{D}$ & Three dimensional \\
\hline$A_{0}$ & Stroke amplitude \\
\hline$A R$ & Aspect ratio \\
\hline$c$ & Chord length \\
\hline$C_{D}$ & Drag coefficient \\
\hline$C_{L}$ & Lift coefficient \\
\hline$C_{P}$ & Aerodynamic power coefficient \\
\hline$\overline{C_{D}}$ & Cycle-average lift coefficient \\
\hline$\overline{C_{L}}$ & Cycle-average lift coefficient \\
\hline$\overline{C_{P}}$ & Cycle-average aerodynamic power coefficient \\
\hline$\overline{C_{S P}}$ & Cycle-averaged specific power coefficient \\
\hline CFL & Courant-Friedrichs-Lewy \\
\hline$\Omega_{\max }^{*}$ & Steady state angular velocity \\
\hline $\operatorname{Re}$ & Reynolds number \\
\hline$k$ & Reduced frequency \\
\hline$f$ & Flapping frequency \\
\hline
\end{tabular}




\begin{tabular}{|c|c|}
\hline$F_{D}$ & Drag force \\
\hline$F_{L}$ & Lift force \\
\hline$v$ & Kinematics viscosity \\
\hline$R_{75}$ & Distance from rotational center to $75 \%$ chord in span-wise \\
\hline r.m.s. & Root-mean-square \\
\hline$S$ & Area of plate surface \\
\hline$T E F$ & Trailing-edge flap \\
\hline$U_{r e f}$ & Reference velocity \\
\hline$U_{\max }$ & Maximum velocity \\
\hline$\theta_{L}$ & Leading-edge rotational angle \\
\hline$\theta_{T}$ & Trailing-edge rotational angle \\
\hline$\beta_{L}$ & Leading-edge rotational amplitude \\
\hline$\beta_{T}$ & Trailing-edge deflection amplitude \\
\hline$\varphi$ & $\begin{array}{l}\text { Phase shift between leading-edge rotation and trailing-edge } \\
\text { deflection }\end{array}$ \\
\hline$\eta_{\text {aero }}$ & Aerodynamic efficiency \\
\hline$r_{\text {root }}$ & Distance from the root plate to the rotational center \\
\hline$\Psi$ & Azimuthal angle \\
\hline$\Psi_{m}$ & Azimuthal angle amplitude \\
\hline$\phi_{\Psi}$ & Azimuthal angle phase offset \\
\hline
\end{tabular}


$\Theta$

$\Theta_{m}$

$\phi_{\Theta}$

$\Theta_{0}$
Pitching angle

Pitching angle amplitude

Pitching angle offset

Initial pitching angle 


\section{Chapter 1: Introduction}

There is a serious effort to design aircraft that are as small as possible for advance surveillance and detection missions. These aircraft, called micro air vehicles (MAVs), are normally compact with a $20 \mathrm{~cm}$ maximum dimension and entire payload mass of $200 \mathrm{~g}$ or less, and which can flight at speeds between 10 and $15 \mathrm{~m} / \mathrm{s}$. At this relatively low Reynolds numbers $(10,000-100,000)$ regime, the aerodynamic efficiency (lift-to-drag ratio) of conventional fixed airfoils rapidly deteriorates at $\operatorname{Re}<100,000$ as shown in Figure 1 from McMasters and Henderson [1]. The chief reason for the deterioration in the performance is that at low Reynolds numbers, the boundary layer remains laminar downstream of the location of minimum pressure on the airfoil making it very susceptible to flow separation as the angle of attack increases resulting in an early onset of stall [2]. In addition, because of the low aspect ratio wings used in MAVs, the tip vortex covers a major part of the wing and the aerodynamic performance is affected greatly by the shedding of the tip vortices [3].

Small birds and insects whose flight regime coincides with that of MAVs use flapping wings to provide both lift and thrust to overcome the deteriorating performance of fixed wings. They do this by taking advantage of unsteady flow mechanisms using wing kinematics evolved over millions of years. The kinematics of wing motion is often a complex combination of translation and rotation in the stroke plane with significant morphological changes for optimal flow control. Hence understanding the unsteady aerodynamics of flapping wings is critical to the design of efficient MAVs. Whereas it may not be possible to mimic the exact nature of bird and insect flight in its full natural complexity, understanding the major unsteady flow mechanisms employed for efficient flight and translating those to simple kinematics is required. 


\subsection{Unsteady Aerodynamics of Flapping Flight}

The unsteady aerodynamic phenomena that allow insects to operate efficiently at small scales are produced by dynamic rotations of the insect wing. These wing rotations are oscillatory in nature and encompass a large variety of motion profiles and associated tip paths for different species of insects. The tip path associated with a particular insect species depends on the insect morphology, the configuration of the wing and body structures and joints, and the insect physiology which determines how the wing is actuated.

At wing lengths on the order of 50 millimeters, the dominant unsteady aerodynamic phenomenon assumed to be responsible for aerodynamic lift is a leading edge vortex (LEV) produced by a laminar flow separation near the leading edge of the wing. This vortical structure produces a region of low pressure near the wing surface and influences the strength of the bound circulation about the wing. This LEV is similar to the vortical structure produced during dynamic stall observed for conventional configurations undergoing rapid pitch or plunge maneuvers. Unlike the vortex produced during a dynamic stall, the leading edge vortex observed for applying wing fliers is stable for the duration of the half-stroke.

In addition to circulatory aerodynamic phenomena, there are aerodynamic forces associated with the acceleration of the volume of air in the immediate vicinity of the wing as the wing changes direction of motion at the end of each half-stroke. This noncirculatory phenomenon influences the pressure distribution along the wing surface as well as the pressure and velocity field about the wing. The circulatory and noncirculatory aerodynamic phenomena each produce localized wake structures. These local 
wake structures interact to form the complex wake structures observed for natural fliers. Sustained flight is achieved when the vortical wake structures align in space and time to form a coherent momentum jet.

\subsection{Dynamic Deformed Wing in Flapping Flight}

Despite the unsteady aerodynamic phenomenon, another key feature of natural flapping fliers is wing flexibility. Natural flyers typically have flexible wings to adapt to the flow environment (as shown in Figure 2). Birds have different layers of feathers and often connected to each other. Hence, they can adjust the wing plan-form for a particular flight mode. Bats have more than two dozen independently controlled joints in the wing and highly deforming bones that enable them to fly at either a positive or a negative angle of attack, to dynamically change wing camber. Insect wings differ from those of vertebrates in that they lack internal musculature extending into the aerodynamic surface of the wing. Thus, insects have little active control over wing properties, and most deformations are a product of the passive mechanical properties of the wing interacting with the inertial and aerodynamic forces it generates while flapping. It is widely thought wing deformation, either active or passive, would potentially provide new aerodynamic mechanisms of aerodynamic force productions over completely rigid wings in flying.

By applying either a two-dimensional foil or a highly idealized three-dimensional wing model $[4,5,6]$, recent studies on the dynamic deformations during flapping flight mainly focused on the negative camber resulting from the aerodynamic and inertial forces. The development of high-speed photogrammetry made it is possible for detailed measurements of wing deformation during high frequency flapping motion. The study of 
deformable wing kinematics of locust [7] used a large number of marker points approximately 100 per wing shows that both fore- and hind- wings were positively cambered on the down-stroke through an 'umbrella effect' whereby the trailing edge tension compressed the wing fan corrugated, reducing the projected area by 30 per cent, and releasing the tension in the trailing edge. The high-fidelity 3-D dragonfly wing surface reconstruction made by Koehler et. al [8] show that wing presents up to $15 \%$ positive chord-wise camber. Birds and bats can also actively change the span by flexing their wings to form camber. However, bats and birds flex their wings in different manners. The wing-surface area of a bird's wing consists mostly of feathers, which can slide over each other as the wing is flexed and still maintain a smooth surface. Bat wings, in contrast, are mostly thin membrane supported by the arm bones and the enormously elongated finger bones. Given the stretchiness of the wing membrane, bats can flex their wings a little, reducing the span by about $20 \%$, but they cannot flex their wings too much or the wing membrane will go slack. Slack membranes are inefficient, because drag goes up, and the trailing edges are prone to flutter, making them more difficult for fast flight[9].

\subsection{Project Overview}

To study the deformable flapping wing aerodynamics, a simplified two-link plate model is used in the current work. The leading-edge portion of the plate is driven by a prescribed hovering motion moving in a horizontal stroke plane. The deflection of trailing-edge flap follows a sinusoidal function with respect to the leading-edge for mimicking time-varying camber deformation. Key parameters for determining deformed plate kinematics are studied. The overall goal of this thesis is to investigate the 
aerodynamic performance and unsteady flow formation of a hovering flapping plate by adding an articulated trailing-edge flap, in both two-dimensional and three-dimensional. An in-house sharp-interface immersed-boundary method has been used to simulate the unsteady flow. Results from current parametric studies will be used to analyze unsteady force productions due to dynamic trailing-edge flap in hovering motion.

\subsection{Organization of Thesis}

Chapter 2 provides a description of numerical methods applied in current simulations. The solver validations are also conducted by comparing experimental measurement in both translational and rotational motion. Chapter 3 examines the influence of key kinematics parameters, such as trailing-edge deflection amplitude and phase shift, on the unsteady aerodynamic phenomena in two-dimensional. Chapter 4 further explores the aerodynamic performances and flow modulations in threedimensional. The effects such as aspect-ratio and geometry of trailing-edge flap will be studied. Chapter 4 examines the influence of key kinematics parameters on the unsteady aerodynamic phenomena in three-dimensional. Chapter 5 summarizes the influence of the kinematic parameters on the unsteady aerodynamic mechanisms and presents recommendations for future research efforts. 


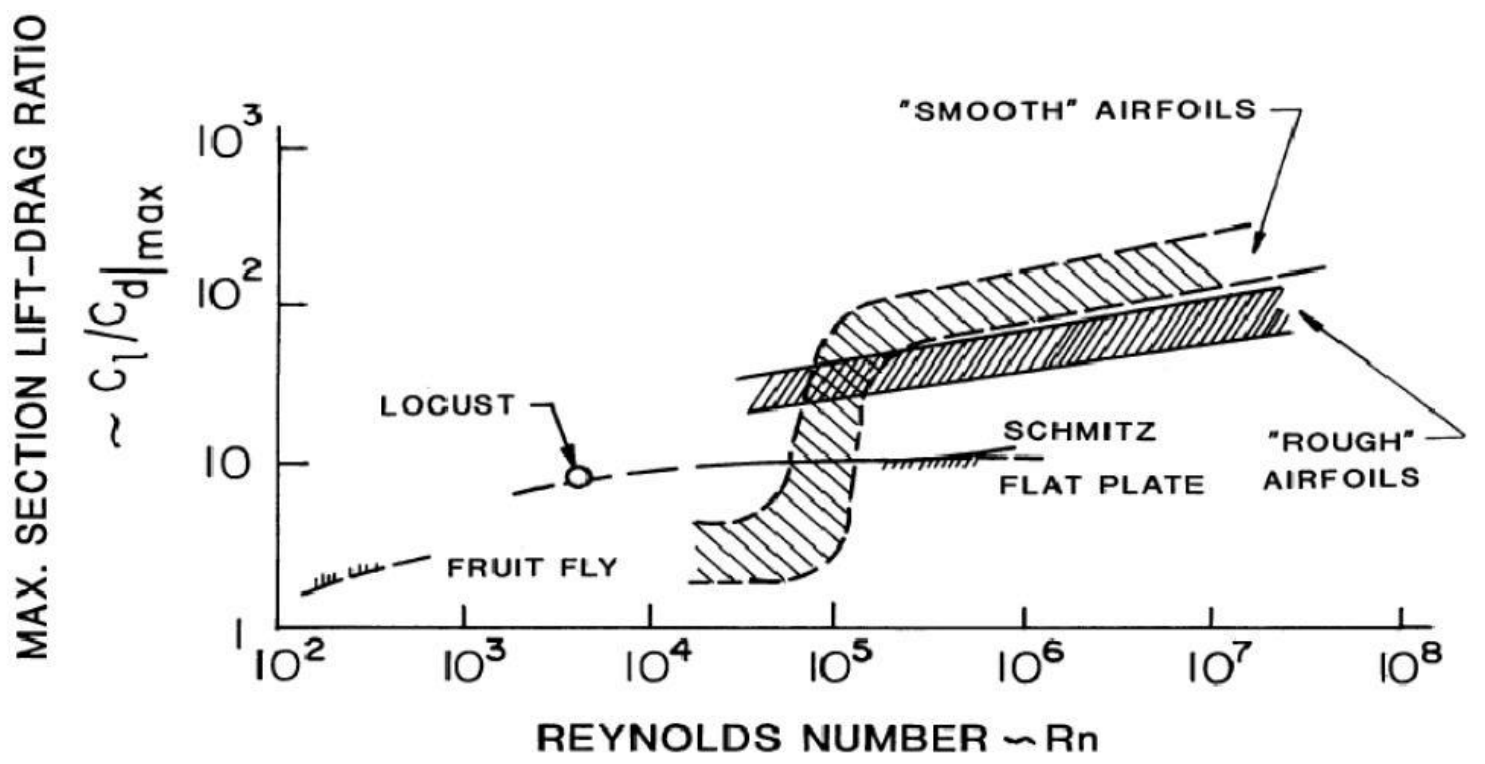

Figure 1: Variation of aerodynamic efficiency with Reynolds number. The aerodynamic efficiency of the smooth airfoil drops rapidly when Reynolds number is less than 100,000 (From Mcmasters, J. H. and Henderson, 1980[1]) 

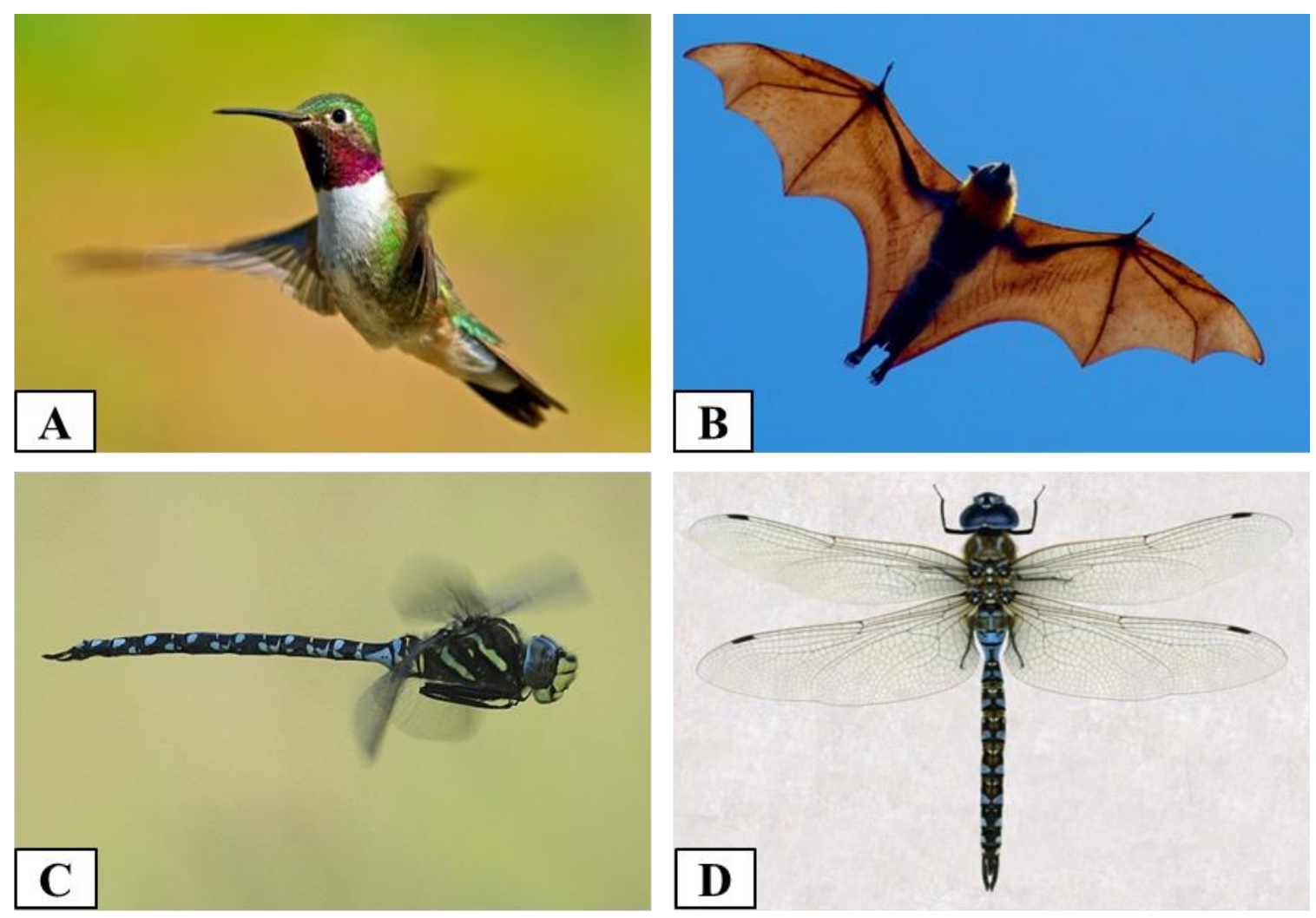

Figure 2: (A) Hummingbird hovering flight, picture courtesy of J. M. Hughes; (B) Flying bat, picture courtesy of Duncan PJ; (C) Dragonfly in free flight showing large scale wing deformation, picture courtesy of H. Alberts; (D) Veinous wing structure of dragonfly, picture courtesy of F. L. Mitchell. 


\section{Chapter 2: Computational Tool}

A second-order finite-difference based solver [10] for simulating flows with immersed boundaries on fixed Cartesian grids has been developed which allows us to explore the wake structures with complex immersed three-dimensional moving bodies. The biggest advantage of this method is that a Cartesian grid method wherein flow past immersed complex geometrics can be simulated on non-body conformal Cartesian grids and this eliminates the need for complicated re-meshing algorithms. The Eulerian form of the Navier-Stokes equations is discretized on a Cartesian mesh and boundary conditions on the immersed boundary are imposed through a "ghost-cell" procedure. The method also employs a second-order center-difference scheme in space and a second-order accurate fractional-step method for time advancement. The pressure Poisson equation is solved using the semi-coarsening multi-grid method with immersed-boundary methodology. The details of this method and validation of the code can be found in [11].

\subsection{Numerical Method}

The equations governing the flow in the numerical solver are the time-dependent, viscous incompressible Navier-Stokes equations, written in indicial form as:

$$
\begin{gathered}
\frac{\partial u_{i}}{\partial x_{i}}=0 \\
\frac{\partial u_{i}}{\partial t}+\frac{\partial\left(u_{i} u_{j}\right)}{\partial x_{j}}=-\frac{1}{\rho} \frac{\partial P}{\partial x_{i}}+v \frac{\partial}{\partial x_{j}}\left(\frac{\partial u_{i}}{\partial x_{j}}\right)
\end{gathered}
$$

where the indices, $\mathrm{i}=1,2,3$, represent the $\mathrm{x}, \mathrm{y}$, and $\mathrm{z}$ directions, respectively; and the velocity components $u_{1}, u_{2}$, and $u_{3}$ corresponding to $\mathrm{u}, \mathrm{v}, \mathrm{w}$ respectively; $\rho$ is density 
and $v$ represents kinematic viscosity. The equations are non-dimensionalized with the appropriate chord length of forewings and free stream velocity. Hence, the tensor expression in equation (2) is written as:

$$
\frac{\partial u_{i}}{\partial t}+\frac{\partial\left(u_{i} u_{j}\right)}{\partial x_{j}}=-\frac{\partial P}{\partial x_{i}}+\frac{1}{\operatorname{Re}} \frac{\partial^{2} u_{i}}{\partial x_{j} \partial x_{j}}
$$

Where, Re corresponds to Reynolds number.

The non-dimensional form of the Navier-Strokes equations is discretized using a cell-centered, collocated (non-staggered) arrangement where all variables (i.e., velocity components and pressure) are located at the same physical location. The equations are integrated in time using the fractional step method [12], with the first step consisting of advancing the solution from time level " $\mathrm{n}$ " to " $\mathrm{n}+1$ " through an intermediate advection diffusion step, where the momentum equations without the pressure gradient term are calculated to obtain an intermediate velocity field. After the intermediate velocity is obtained, a second-order Adams-Bashforth scheme is implemented to discretize the convective terms while the diffusion terms are discretized with an implicit CrankNicolson technique which eliminated the viscous stability constraint. The second step of the fractional step method is the solution of a pressure correction equation by solving a Poisson equation. A Neumann boundary condition is implemented on this pressure correction step at all boundaries. The Poisson equation being the most time consuming part of the solution algorithm, is solved with a flexible and efficient nonstandard geometric multi-grid algorithm with a flexible semi-coarsening strategy [13] which employs a Gauss-Siedel liner-SOR smoother. Performance tests of the multi-grid method have been carried out by $[14,15]$. 
The immersed boundary method is an innovative approach in simulating fluid flow over bodies which are imbedded within a Cartesian grid. The key concept of the immersed boundary method is to compute the flow variables for the ghost-cells (GC), such that boundary conditions on the immersed boundary in the vicinity of the ghost-cells are satisfied while preserving second-order accuracy. Ghost-cells are those cells whose centers lie inside the immersed body and have at least one neighboring cell which lies outside the immersed body. The process begins by developing the immersed boundary comprised of a number of densely spaced marker points connected by linear segments. A non-conformal Cartesian grid is then generated followed by a procedure that identifies the fluid-cells and solid-cells. Fluid-cells are cells whose cell centers line outside of the body, and solid-cells are made up of the remaining cells with cell centers inside the body that are not adjacent to the immersed boundary.

\subsection{Validations}

In order to demonstrate the validity of the numerical code, simulations of rectangular plat with aspect ratio $(A R)$ two was performed in different types of kinematics profile and compared to the published results and experimental measurements. Overall, our simulation results showed a pretty well agreement with all the experimental works, and more detailed described as following subsections.

\subsubsection{Flow Pass Stationary Plate}

In the first case, simulations of rectangular plate $\left(\mathrm{AR}=2, \mathrm{AOA}=30^{\circ}, \mathrm{Re}=300\right)$ was performed and compared to the published results of Taira and Colonius [16]. The wake 
structure of rectangular plate and time trace of lift and drag coefficients at large time are selected to compare.

Our simulations were performed on a $200 \times 88 \times 128$ (grid size of dense domain) nonuniform Cartesian grid with a domain size $10.1 \times 10 \times 10$ with the smallest resolution of $\Delta x=0.02$. Grid stretching is applied in all directions with finer resolution near the plate to capture the wake structure. The computational domain used in the validation case is matched with the original setup.

In Figure 3, the comparison of lift and drag coefficients are represented. The results of interest in this particular validation case were the time trace of lift and drag coefficients. The blue lines represent our simulation results and red delta shows the digitized data from publication results. In our simulation we also observe the same phenomenon [16] that lift and drag reach to a stable steady state after the initial transient generated by the impulsive start settles down.

From Figure 4, it can be seen that the formation and evolution of leading-edge and tip vortices are in good agreement between the publication and our simulation results despite the difference of far field vortices structure and the velocity profile. Our simulation is able to capture even the thin layer of far field vortex sheet emanating from the leading edge, which would correspond to the more dense stretching grids we used behind the dense domain. 
A

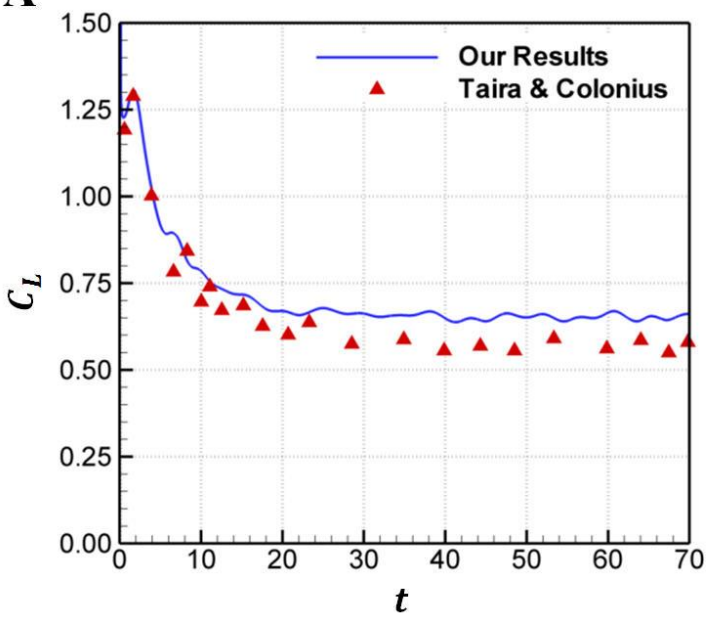

B

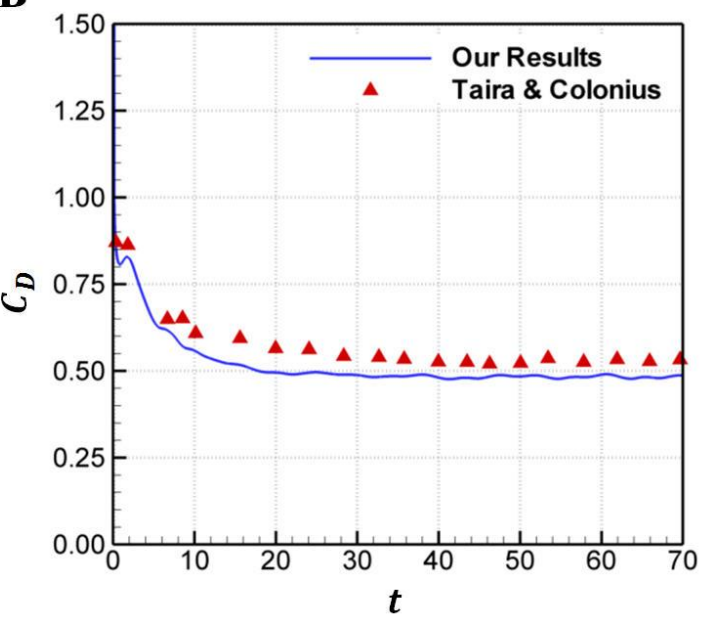

Figure 3: Time history of lift and drag coefficients for rectangular plan-form at $\mathrm{Re}=300$, and $\boldsymbol{\alpha}=3 \mathbf{0}^{\circ}$. (A) Comparison of Lift coefficient; (B) Comparison of Drag coefficient.

The domain dimension and the number of grid points (especially for grid stretching part) were determined without performing detailed domain independence and grid refinement studies. This may cause our lift and drag coefficient exist a few difference when the flow achieved to a steady state. This maybe also the reason that our far flow wake structure exist a litter bit difference compared with the publication results. Regardless of this fact, the results obtained are in good agreement with those of Taira and Colonius [16]. 

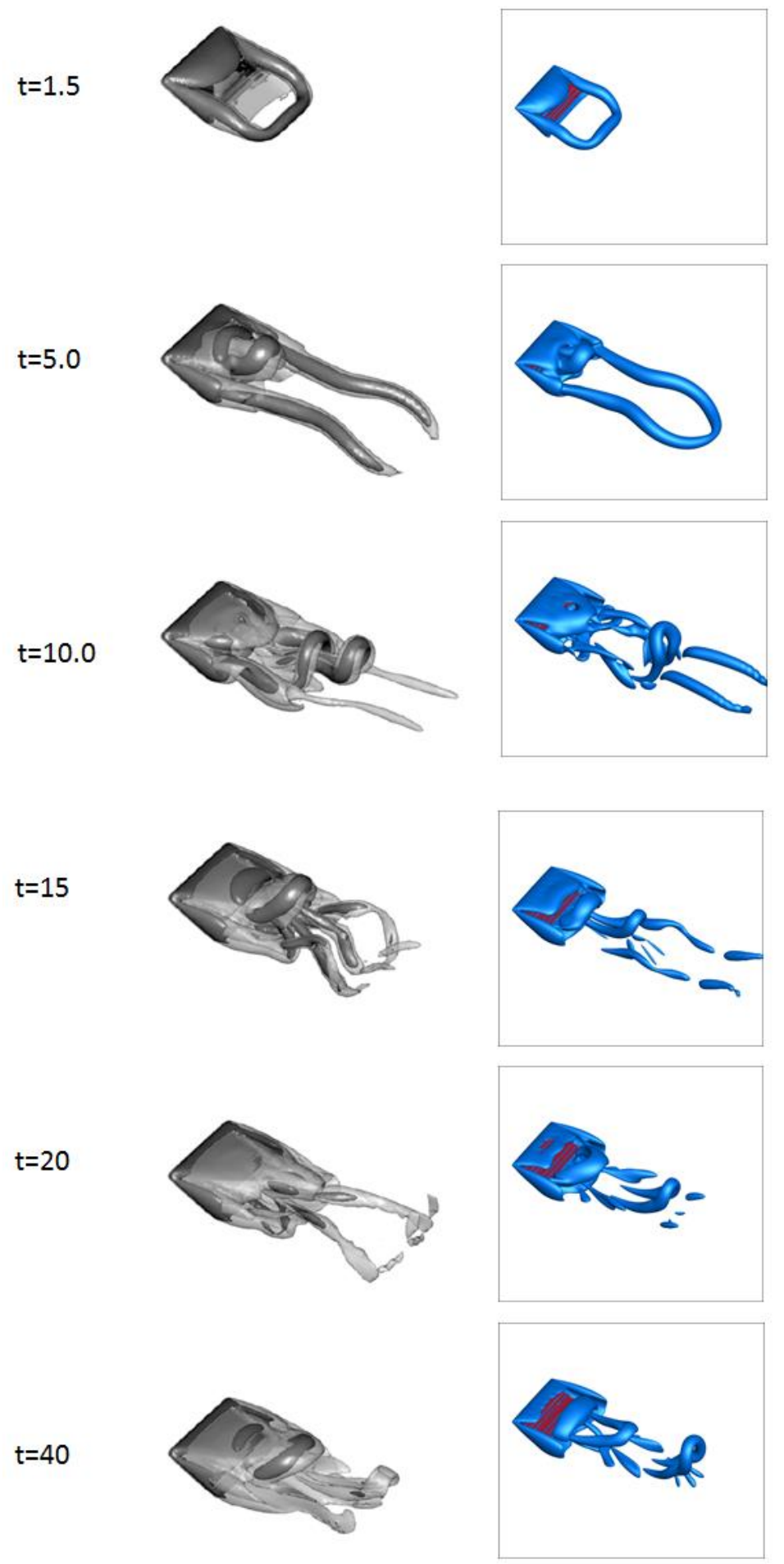

(A)

(B)

Figure 4: Wake vortices behind a rectangular wing of $\mathrm{AR}=2$ at $\alpha=30^{\circ}$ and $\mathrm{Re}=300$. Shown are the iso-surface of $\mathrm{Q}=3$. (A) from [16]; (B) our simulation results. 


\subsubsection{Translational Plate}

In the second case, results from the three-dimensional simulations was compared with measurements from an oil tank experiment $[17,18]$ of flows over a rectangular plate of dimension $80 \mathrm{~mm} \times 160 \mathrm{~mm} \times 3 \mathrm{~mm}(A R=2)$. The temperature in the laboratory is regulated at $20 \pm 1{ }^{\circ} \mathrm{C}$ such that there is less than $3 \%$ variation in the kinematic viscosity $(12 \pm 2 \mathrm{cSt})$. The rectangular flat plate of angle of attack at $35^{\circ}, 45^{\circ}$, and $55^{\circ}$ was accelerated and reached to its maximum velocity after 0.5 seconds. The plate translation configuration and velocity profile are shown in Figure 5. For all cases, the Reynolds number is fixed at 800 .
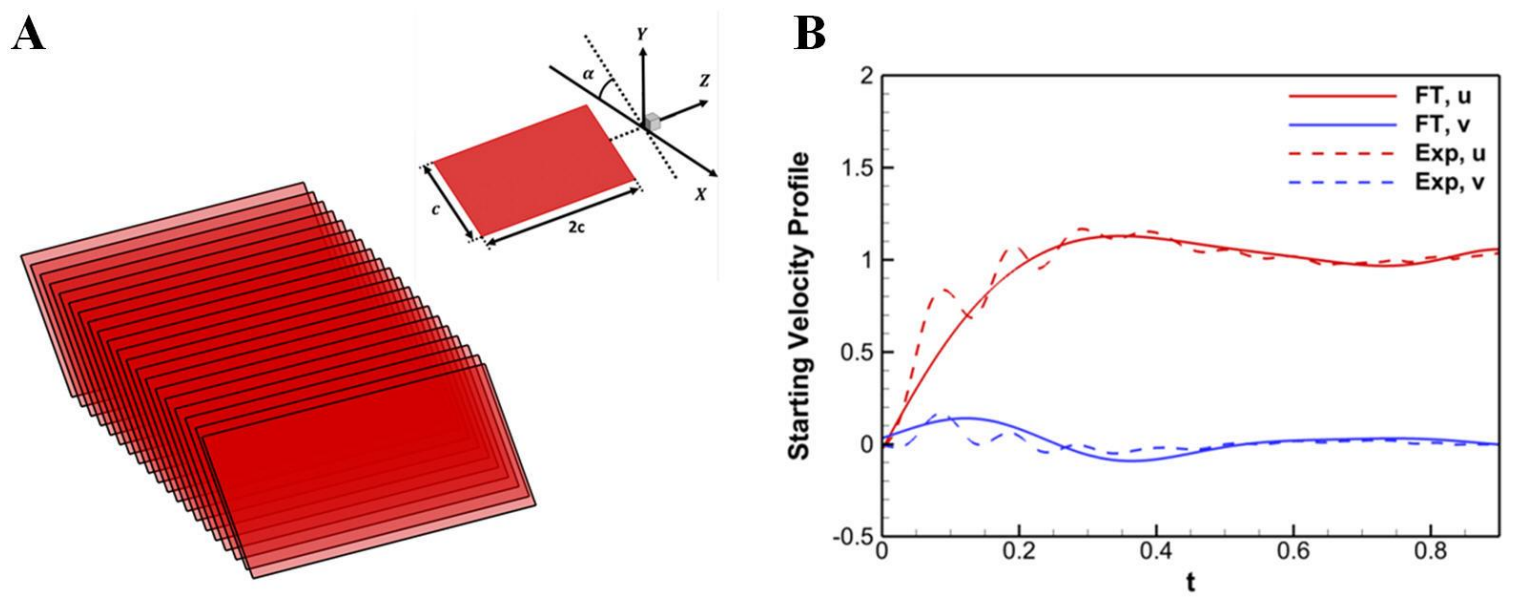

Figure 5: Rectangular plate $(A R=2)$ translation configuration (A) and velocity profile in time (B).

Simulations are performed on a $279 \times 171 \times 279$ non-uniform Cartesian grid with a domain size $18 \times 12 \times 18$ with the smallest resolution of $\Delta x=0.015$. Grid stretching is applied in all directions with finer resolution near the plate to capture the wake structure. The comparison of force history presented in Fig shows a good agreement. The time of 
maximum lift appeared at $\mathrm{t} \approx 0.3$, which results from the inertial force during impulsive start process.

A

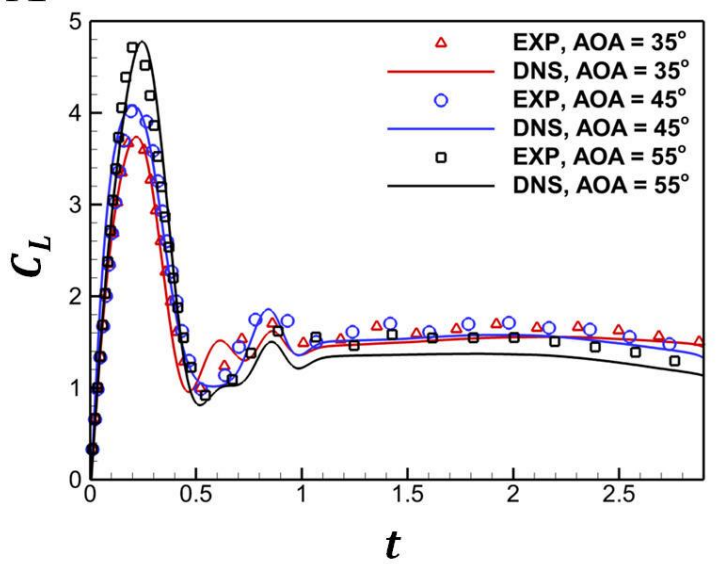

B

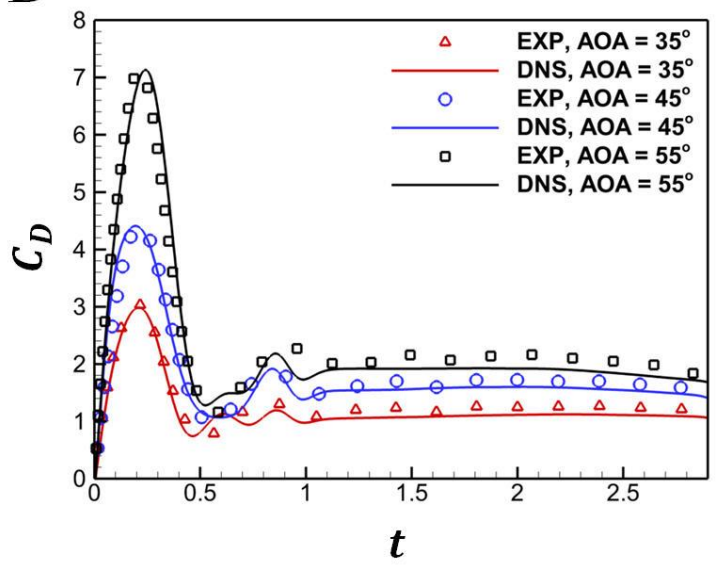

Figure 6: Time history of lift and drag coefficients for rectangular plan-form $(A R=2)$ in

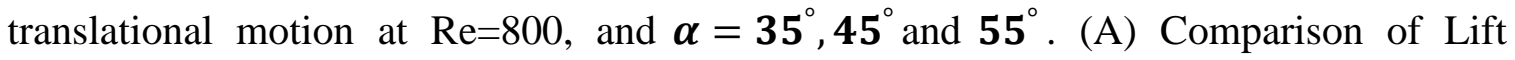
coefficient; (B) Comparison of Drag coefficient.

\subsubsection{Rotational Plate}

For the third validation case, rotational experiment [19] was performed in an 18 in $\times 18$ in $\times 18$ in glass tank filled with mixing water/glycerin. The geometry considered in this study is an aspect-ratio-two rectangular wing. The root of the wing is extended out a distance of $\mathrm{r}=0.5 \mathrm{c}$ from the rotation axis at a fixed angle of attack $45^{\circ}$. The wing is initially at rest in quiescent flow before accelerating to a constant rotational rate as it revolves around its axis, and the total rotational angle is $720^{\circ}$. The velocity profile used for the experiments consists of a piecewise linear function in three phases: an acceleration phase, a constant speed phase, and a deceleration phase. The acceleration 
and deceleration were chosen so that the speed increases linearly over $0.25 \mathrm{c}$ of travel at $75 \%$ of tip radius. The angular velocity profile is given by Eq. (4) as following:

$$
\Omega^{*}(\tau)=\left\{\begin{array}{lc}
\frac{\Omega_{\max }^{*}}{2 a\left(\tau_{2}-\tau_{1}\right)} \ln \left[\frac{\cosh \left(a\left(\tau-\tau_{1}\right)\right)}{\cosh \left(a\left(\tau-\tau_{2}\right)\right)}\right]+\frac{\Omega_{\max }^{*}}{2}, & 0 \leq \tau \leq \frac{\left(\tau_{2}+\tau_{3}\right)}{2} \\
\frac{\Omega_{\max }^{*}}{2 a\left(\tau_{4}-\tau_{3}\right)} \ln \left[\frac{\cosh \left(a\left(\tau-\tau_{3}\right)\right)}{\cosh \left(a\left(\tau-\tau_{4}\right)\right)}\right]+\frac{\Omega_{\max }^{*}}{2}, & \tau \geq \frac{\left(\tau_{2}+\tau_{3}\right)}{2}
\end{array}\right.
$$

where $\Omega_{\max }^{*}$ is the steady state angular velocity; $a$ is the smoothing parameter, $\tau_{1}$ is the start time, $\tau_{2}$ marks the end of the acceleration, $\tau_{3}$ marks the beginning of the deceleration, and $\tau_{4}$ marks the end of deceleration.
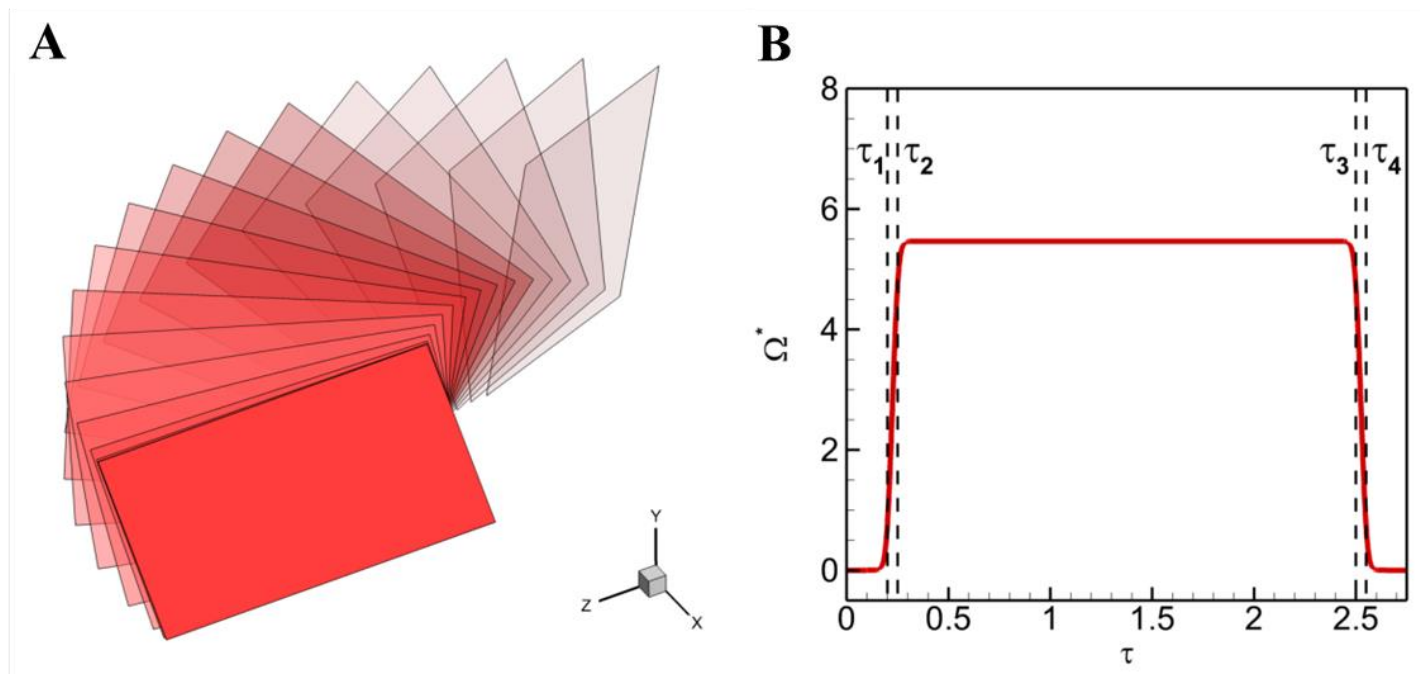

Figure 7: Rectangular plate $(A R=2)$ rotational configuration $(\mathrm{A})$ and angular velocity profile in time (B).

This expression is modified form of a function described by Eldredge et al., [20], which allows for a continuous motion that is sufficiently differentiable, thereby avoiding discontinuities in the angular acceleration. In current validation case, the value of 
smoothing parameter, $a$, is set to 50 . Figure 7 shows the smoothed angular velocity profile. The dashed vertical line marks the acceleration duration $\left(\tau_{2}-\tau_{1}\right)$ and deceleration duration $\left(\tau_{4}-\tau_{3}\right)$.

The Reynolds number is defined as $R e=\Omega_{\text {max }}^{*} R_{75} c / v$, where $\Omega_{\text {max }}^{*}$ is the steady state angular velocity, $R_{75}$ is the representative radius, chosen as $75 \%$ of the tip radius, $c$ is the chord of the wing, and $v$ is the kinematic viscosity of the fluid. The force coefficient is defined as $C_{L, D}=\left(F_{L}, F_{D}\right) / 0.5 \rho\left(\Omega_{\max }^{*} R_{75}\right)^{2} S$, in which $F_{L}$ and $F_{D}$ are lift and drag force, respectively, $\rho$ is the density of the fluid, and $S$ is the area of the wing.

The Reynolds number for this case equals to 500. Lift coefficient time-histories are shown in Figure 8 corresponding to the wing kinematics in Figure 7. For the case with $0.25 \mathrm{c}$ acceleration duration, the lift coefficients experienced a sharp peak associated with the inertial forces, followed shortly by a second peak. The simulation results for this case also have a good agreement with the experimental measurement. 
A

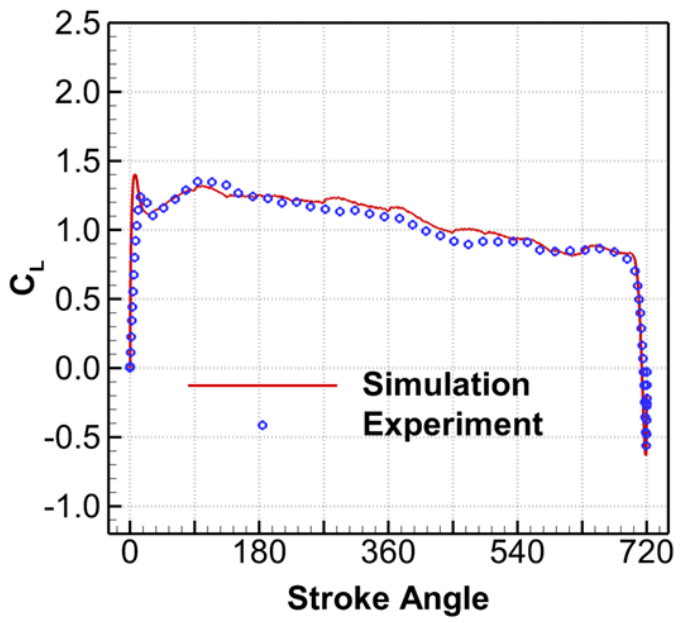

B

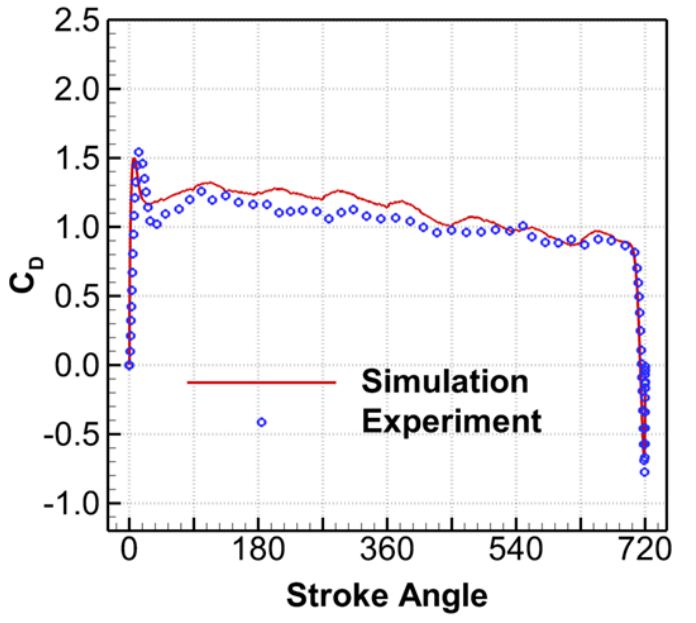

Figure 8: Time history of lift and drag coefficients for rectangular plan-form $(A R=2)$ in rotational motion at $\mathrm{Re}=500$, and $\boldsymbol{\alpha}=45^{\circ}$. (A) Comparison of Lift coefficient; (B) Comparison of Drag coefficient. 


\section{Chapter 3: Parametric Study of Two-Dimensional Hovering Plate with Trailing Edge Flap}

\subsection{Plate Kinematics}

In this study, the deformable wing is simplified as a rigid plate hinged with a controllable trailing-edge flap. It follows a sinusoidal flapping and pitching motion in the Cartesian coordinates system. Specifically, the leading-edge portion of the plate is constrained moving in a horizontal stroke plane according to a prescribed function given by Eq. (5) and (6):

$$
\begin{gathered}
x(t)=\frac{A_{0}}{2}(1+\cos 2 \pi f t), \quad \mathrm{y}(t)=0 \\
\theta_{L}(t)=\beta_{L} \sin (2 \pi f t)
\end{gathered}
$$

Where, $x(t)$ and $y(t)$ denote the position of the leading-edge of the chord, respectively, $\theta_{L}(t)$ is the leading-edge orientation relative to vertical direction, $f$ is the flapping frequency, $A_{0}$ and $\beta_{L}$ are the amplitudes of translation and rotation, respectively.

A $25 \%$ chord length trailing-edge flap hinged with the leading-edge portion. The deflection angle $\left(\theta_{T}\right)$ of trailing-edge flap is prescribed by Eq. (7) with respect to the leading-edge for mimicking time-varying camber deformation. The plate kinematics can be visualized in Figure 9. The gray chord lines indicate the plate without trailing-edge deflection motion, and the red color chord lines present the deformable plate kinematics.

$$
\theta_{T}(t)=\beta_{T} \sin (2 \pi f t+\varphi)
$$

Where, $\beta_{T}$ is the amplitude of deflection, and $\varphi$ is the phase shift between the leadingedge rotation and trailing-edge deflection. 
A

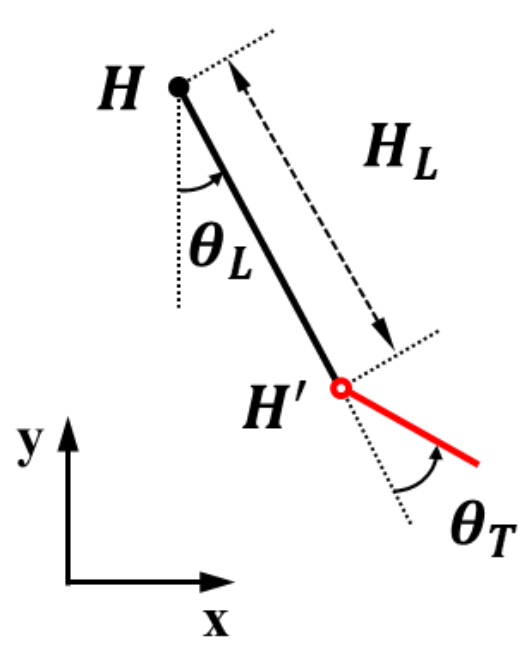

B

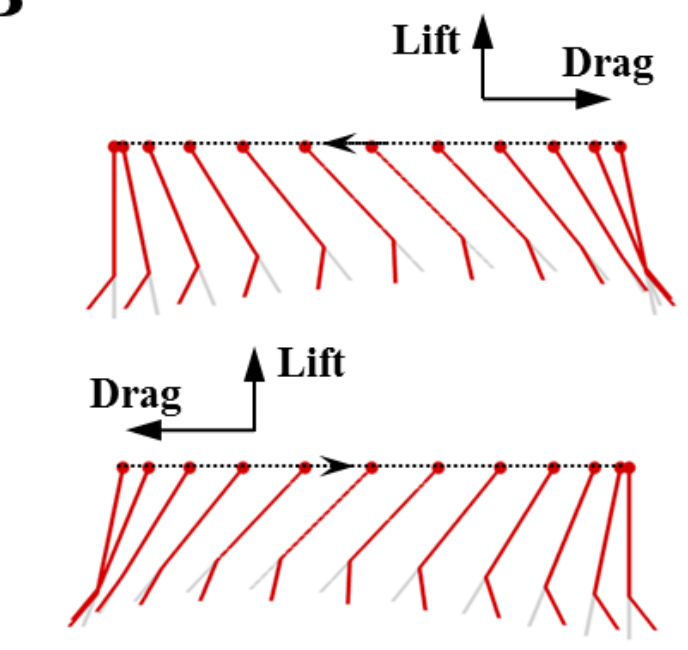

Figure 9: (A) Rigid plate hinged with a $25 \%$ chord length trailing-edge flap, $H$ is the lending-edge, $H_{L}$ denotes the length of leading-edge $\left(H H^{\prime}\right)$ which is $75 \%$ of total chord length; (B) Flapping plate configuration with $A_{0}=3.0, \beta_{T}=60^{\circ}$ and $\varphi=60^{\circ}$.

\subsection{Normalization and Choice of Parameters}

The translational motion of the plate is completely specified by two dimensionless parameters, the Reynolds number ( $R e$ ), and Reduced frequency ( $k$ ), given by Eq. (8) and (9):

$$
\begin{gathered}
R e=\frac{U_{r e f} c}{v}=\frac{\pi f A_{0} c}{v} \\
k=\frac{\pi f c}{U_{r e f}}=\frac{c}{A_{0}}
\end{gathered}
$$

Where, $U_{r e f}$ is the reference velocity and $c$ is the chord length. In current study, the maximum plate velocity of leading-edge is used as the reference velocity, written as $U_{r e f}=\pi f A_{0}$. 
The Reduced frequency $(k)$ provides a measure of unsteadiness associated with a flapping wing via spatial wavelength of the flow disturbance with the chord length. Note that the reduced frequency is inversely proportional to the flapping amplitude of the wing, and not related to flapping frequency.

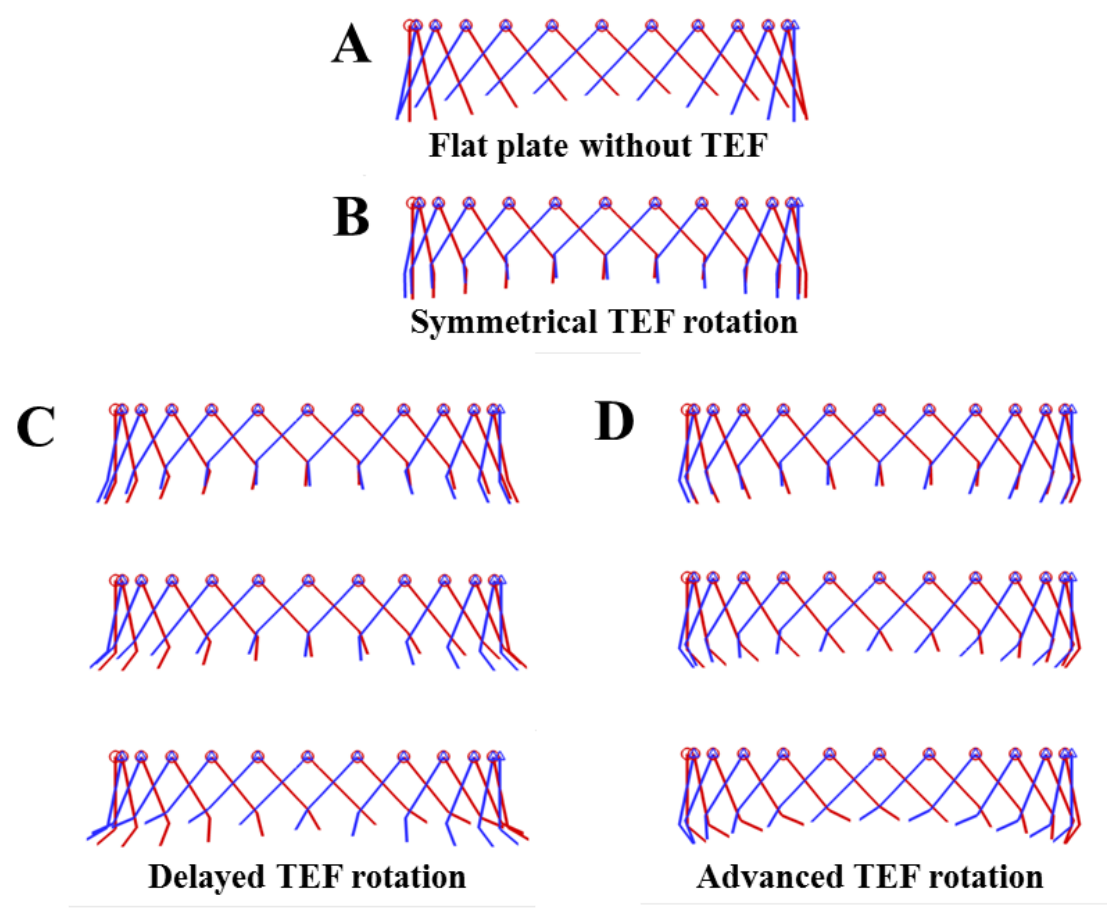

Figure 10: Plate flapping configuration without trailing-edge flap (TEF) (A), with symmetrical $\left(\varphi=0^{\circ}\right)$ TEF rotation (B), with delayed $\left(0^{\circ}<\varphi<180^{\circ}\right)$ TEF rotation (C) and with advanced $\left(-180^{\circ}<\varphi<0^{\circ}\right) \mathrm{TEF}$ rotation. The red and blue color chords indicate the down-stroke and up-stroke, respectively.

The main variables of interest in this study are the trailing-edge deflection amplitude $\left(\beta_{T}\right)$ and phase shift $(\varphi)$ between leading-edge rotation and trailing-edge deflection. The larger deflection amplitude means the more disturbances generated from trailing-edge 
flap. On the other hand, the phase shift corresponds to the phase delay or phase advance of the disturbances. The phase shifts were further characterized as three types, symmetrical deflection $\left(\varphi=0^{\circ}\right)$, advance deflection $\left(-180^{\circ}<\varphi<0^{\circ}\right)$, and delayed deflection $\left(0^{\circ}<\varphi<180^{\circ}\right)$, as shown in Figure 10. In all cases, the Reynolds number $(R e)$ is fixed at 100 , but Reduced frequency ( $k$ ) varies from 0.16 to 0.5 with resulting $A_{0} / c$ from 2 to 6, appropriate for real some insect range, such as fruitflies and bees.

The performance of the flapping plates is to be measured in terms of force (vertical and horizontal) generation and power expenditure. The classical way of assessing and comparing these quantities is through non-dimensionalized coefficients. This nondimensionalization also requires one to choose an appropriate velocity scales as the aforementioned $U_{\text {ref }}$. Then, the force coefficients can be defined as:

$$
\begin{aligned}
C_{L} & =\frac{2 F_{L}}{\rho U_{r e f}^{2} c} \\
C_{D} & =\frac{2 F_{D}}{\rho U_{r e f}^{2} c}
\end{aligned}
$$

Where, $F_{L}$ and $F_{D}$ are the vertical (lift) and horizontal (drag) force, respectively, and $C_{L}$ $C_{D}$ are the corresponding coefficients.

The instantaneous aerodynamic power was calculated as $P=\sum_{i=1}^{n} \vec{F}_{i} \cdot \vec{v}_{i}$, where $\mathrm{n}$ is total number of triangular element on the wing, $\vec{F}_{i}$ is the aerodynamic force on each element and $\overrightarrow{v_{i}}$ is the corresponding velocity of the element. Following a similar approach, the power coefficient can be expressed as: 


$$
C_{P}=\frac{2 P}{\rho U_{r e f}^{3} c^{2}}
$$

Where, $C_{P}$ is the power coefficient and $P$ is the aerodynamic power expended by the plates.

The cycle-averaged aerodynamic efficiency (lift-to-power ratio) is used as a metric of aerodynamic performance in accordance with the ratio of lift-to-drag ratio, which is defined as:

$$
\eta_{\text {aero }}=\overline{C_{L}} / \overline{C_{P}}
$$

As suggested by Wang [21], the cycle-averaged specific power $\left(\overline{C_{S P}}\right)$ based on actuator disk theory [22] was also investigate, which gives a non-dimensional measure:

$$
\overline{C_{S P}}=\frac{\left\langle F_{D}(t) v(t)\right\rangle}{\left\langle F_{L}(t)\right\rangle^{3 / 2}} \sqrt{2 \rho\left(A_{0} / c\right)}
$$

Where, $F_{D}(t)$ and $F_{D}(t)$ are drag and lift force and $v(t)$ is plate velocity.

From these methods and metrics, the deformed plate is simulated, using the details outlined in the following subsections.

\subsection{Computational Setup}

Figure 11(A) shows the flapping plate configuration immersed in the twodimensional non-uniform Cartesian grid. The coordinate directions, which are also shown in Figure 11(B), are as follows: $\mathrm{x}$ is the horizontal direction with $+\mathrm{x}$ pointing towards right side boundary; and y is the vertical direction with $+y$ pointing upward. The flapping 
plates are modelled as zero thickness deformable membrane and the chord length $(c)$ is normalized as one unit.

Figure 11(B) shows the boundary conditions applied on the computational domain boundaries. On all of the boundaries, a far-field boundary condition which amounts to specifying the stream-wise (vertical) velocity component to zero and setting the normal gradients of the other velocity components to zero is applied. The domain size normalized by the chord length $(c)$ is $30 \times 30$ and this large domain ensures that the boundaries do not have any significant effect on the computed results. As shown in Figure 11(A), the grid is designed to provide high resolution in the region around body as well as the wake which is expected to develop below the plates. The final grid adopted here has $289 \times 289$ non-uniform mesh with the smallest resolution of $\Delta x=\Delta y=0.02$. This corresponds to 50 grids across the chord -wise direction on plate surface.

The additional simulations on different grids were also carried out to assess the convergence of the computed flow via both spatial and temporal sensitivity studies discussed in the following subsection. 


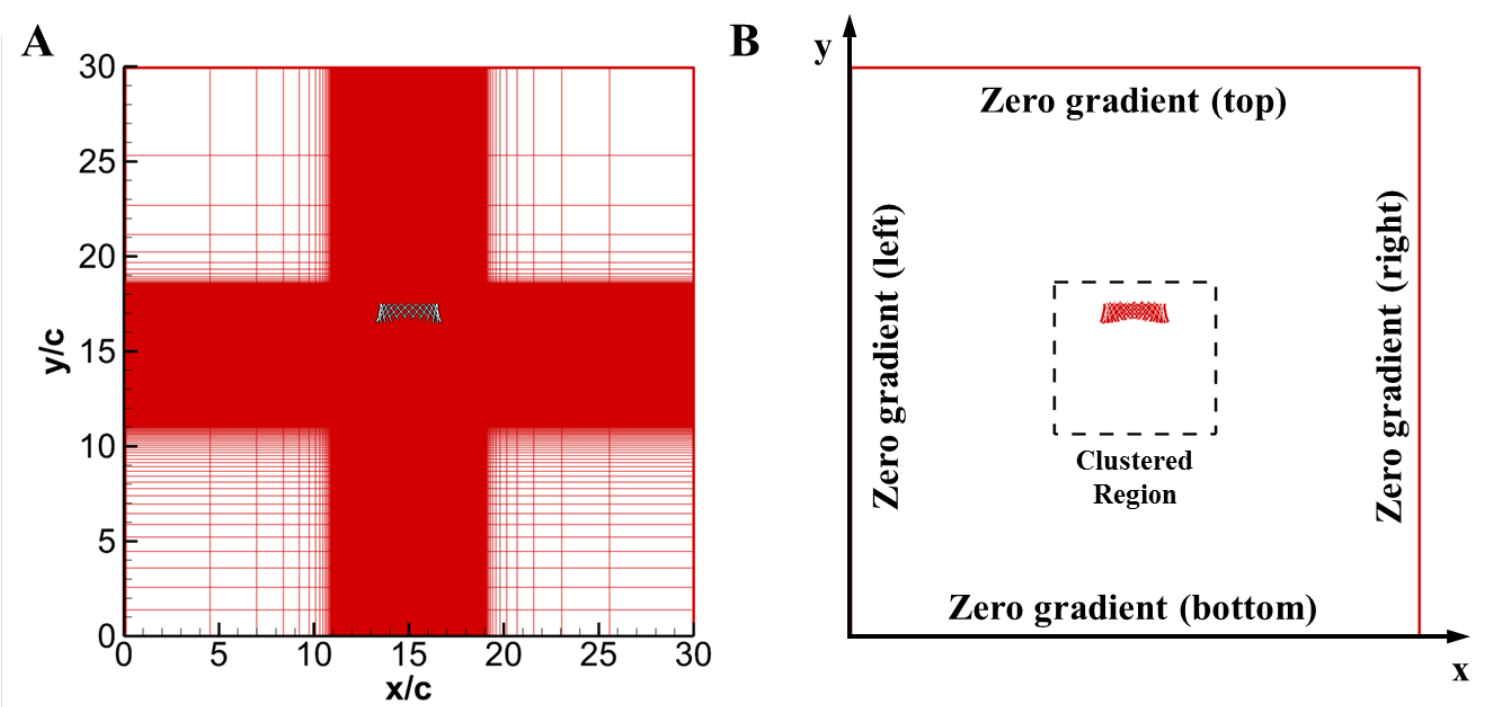

Figure 11: (A) Flapping plate configuration immersed in the two-dimensional nonuniform Cartesian grid; (B) Boundary conditions for the simulation.

\subsection{Spatial and Temporal Sensitivity Study}

In order to identify a suitable number of grid points and time step, grid and time-step sensitivity analyses are performed for the rigid plate with $A_{X} / c=2.0$. Simulation within fine grid was carried out on a grid which had $52 \%$ more grid points than the medium grid, and simulation within coarse grid was carried out on a mesh with $48 \%$ less grid points than the medium grid. Both of these simulations produced a maximum $1 \%$ difference from the medium grid in mean lift and root-mean-square (r.m.s.) values of lift and drag. The temporal sensitivity study also conducted within the medium grid to guarantee the convergence of current simulation. Each simulation was integrated over 10 flapping cycles. Estimates of cycle mean and r.m.s. lift as well as r.m.s. drag indicate that there is a less than a $3 \%$ difference in these quantities from the ninth to the tenth cycle. Thus, the flow quantities reach a near-stationary state by the ninth and tenth cycles and all of the 
flow statistics in the following discussion are estimated based on averaging over the ninth and tenth cycles.
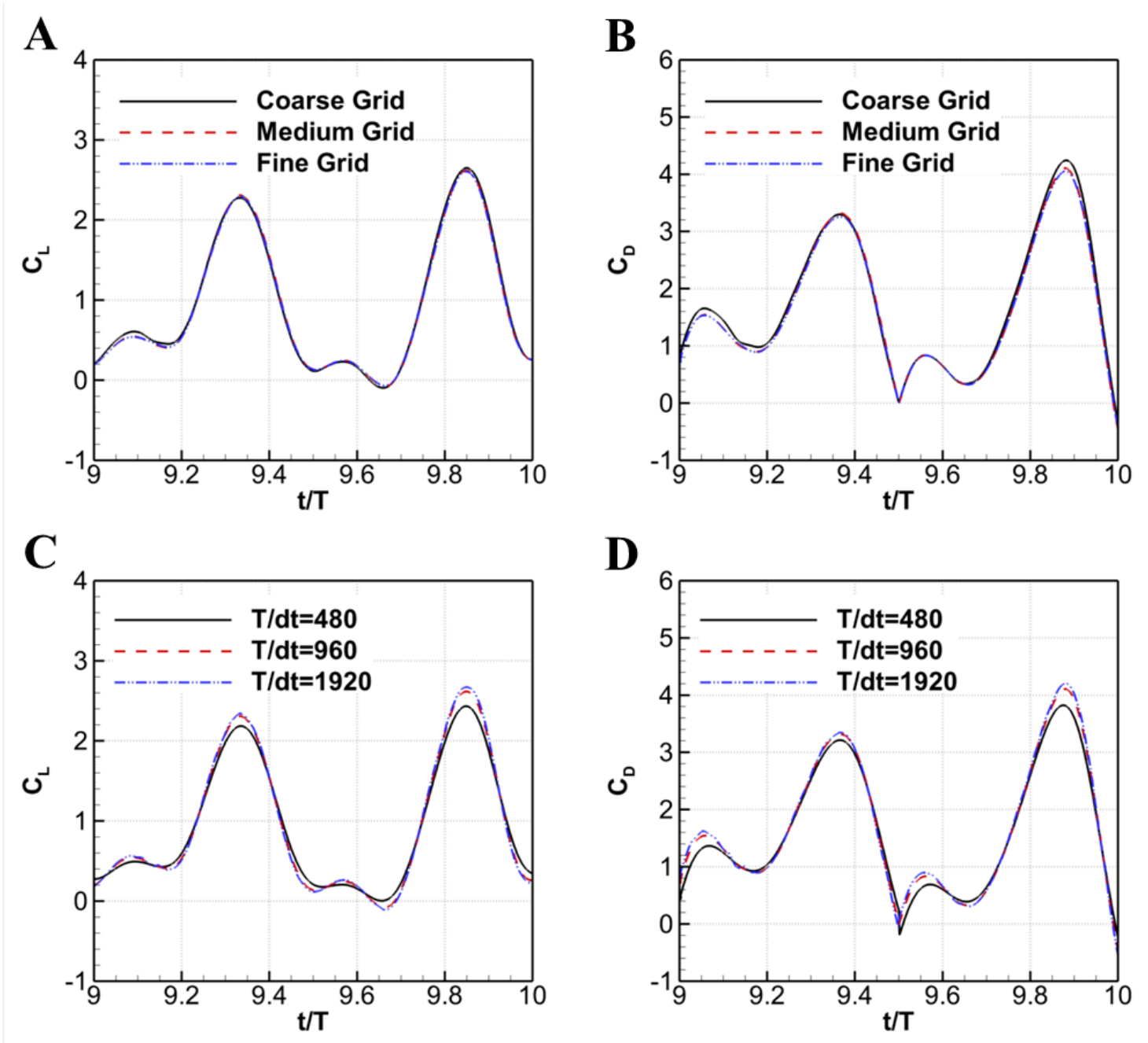

Figure 12: Spatial and Temporal sensitivity analyses for a rigid flapping plate at $A_{0} / c=2.0$. Grid sizes are: Coarse, $0.15 \times 10^{6}$ cells; Medium, $0.29 \times 10^{6}$ cells; and Fine, $0.61 \times 10^{6}$ cells. Lift coefficient $(A, C)$ and drag coefficient $(B, D)$ time history for the $10^{\text {th }}$ flapping stroke. 
From the results shown in Figure 12, the medium grid $\left(0.29 \times 10^{6}\right)$ and $T / d t=960$ are chosen. The time-step chosen for the current study corresponds to a CourantFriedrichs-Lewy (CFL) number of 0.56 .

\subsection{General Performance Comparison}

To provide a more comprehensive picture of how the trailing-edge flap affects the overall unsteady phenomena, I first contrast two special cases with the same flapping stroke amplitude $\left(A_{0} / c=3\right)$. The first case corresponds to a flat plate. The second case corresponds to a rigid plate hinged with a trailing-edge flap, where $\beta_{T}=60^{\circ}$ and $\varphi=60^{\circ}$.

Figure 13 shows a side-by-side comparison of the plate motion, forces, subsequent z-component vorticity snapshots and mean flow in the two cases. The instantaneous forces reach a nearly periodic state after a few strokes. It is not a priori that an arbitrary periodic wing motion coupled to fluid will immediately generate a periodic force because of the complicated interaction with fluids. In our case, the forces can reach an almost periodic state because of negligible interference from vortices generated in previous cycles as seen in Figure 13, which happens only when rotation and translation are phased correctly.

In the case of flap flapping plate, the averaged vertical and horizontal force coefficients are 0.85 and 0.99 , respectively, resulting in a ratio of 0.86 . By contrast, the force generation of the flapping plate with trailing-edge flap enhanced during both downstroke and up-stroke, in which the lift and drag coefficients are 0.99 and 1.38, respectively, resulting in a ratio of 0.71 , which is $17 \%$ less than the flat plate. This 
indicates that the enhancement by adding a trailing-edge flap comes at a cost of lower efficiency.

Comparing the vorticity field in the two cases shows a faster down-ward jet produced by the flapping plate with trailing-edge flap. Figure 13(Biv) shows the timeaveraged velocity below the wing. The velocity is plotted in physical space. The flat plate generates a jet whose width is comparable to the flapping amplitude and it generates down for 4 to 5 chords. By contract, the plate with trailing-edge flap generates a jet whose width is comparable to the chord, and it penetrates downward for more than 7 chords. This difference may be significant when the wing is hovering above a surface, where the ground effect is non-negligible. 
Ai
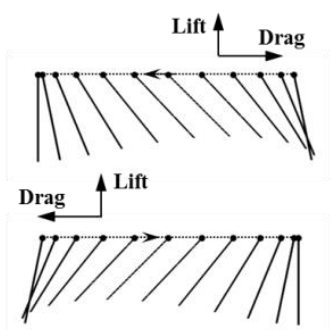

Aii

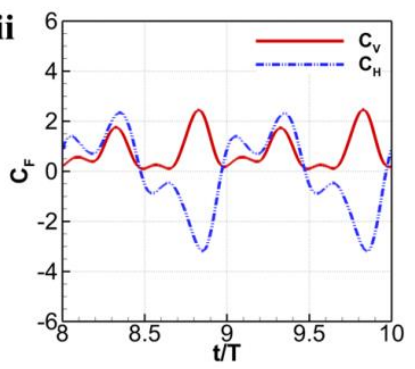

Aiii
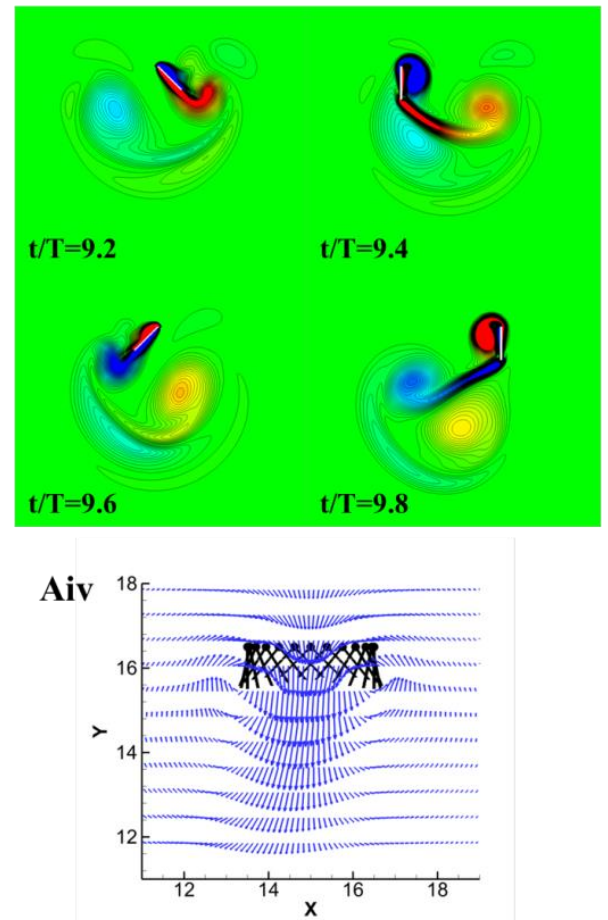

Bi
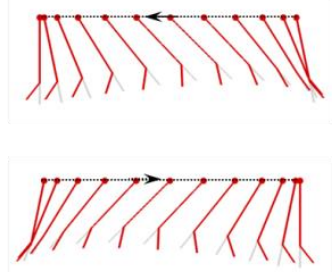

Bii

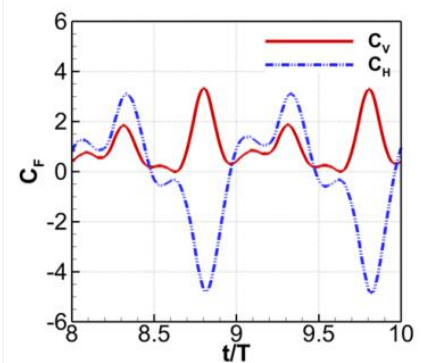

Biii

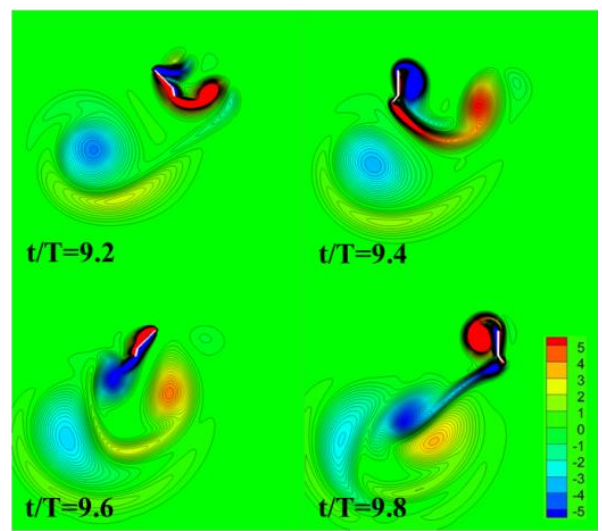

Biv

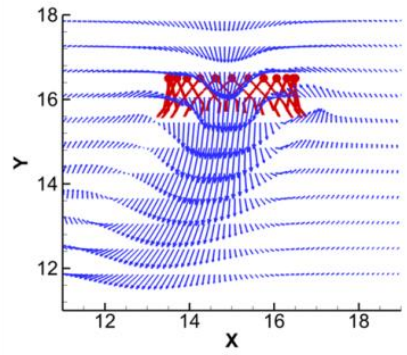

Figure 13: Comparison of idealized normal hovering for rigid plate (A) and hinged plate (B). (i) Wing motion; (ii) instantaneous vertical $\left(\boldsymbol{C}_{\boldsymbol{V}}\right)$ and horizontal $\left(\boldsymbol{C}_{\boldsymbol{H}}\right)$ force coefficients; (iii) snapshots of vorticity (red, counterclockwise rotation; blue, clockwise rotation) during the tenth period, and (iv) time-averaged velocity field. 


\subsection{Stroke Amplitude Effects}

Stroke amplitude is one of the most important kinematics parameters in insect flight. In current two-dimensional work, the stroke amplitude is defined as $A_{0} / c$, which equals the inverse of the Reduce frequency $\left(k=\frac{\pi f c}{U_{r e f}}=\frac{c}{A_{0}}\right)$. The study range of $A_{0} / c$ varies from 2 to 6 according to the reality of insect flapping motion in relatively low Reynolds number $\left(O \sim 10^{2}\right)$ flow [23]. This results reduced frequency $(k)$ changing from 0.17 to 0.5. Normally, $0<k<0.03$ is considered as "quasi-steady" (wake effects are not very significant), $0.03<k<0.1$ can be considered "quasi-unsteady" (wake effects are significant but apparent-mass acceleration effects are negligible), and beyond that, flow is considered "fully unsteady" (all unsteady effects are important) [24]. Hence, we can see that the unsteady aerodynamics play an important in present study.

To elucidate the effect of stroke amplitude $\left(A_{0} / c\right)$, instantaneous lift and drag coefficients of flat plate without trailing-edge flap are first investigated and shown in Figure 14. From this figure, we can observe at least two interesting things. First, as the stroke range increases, both peaks and cycle-averaged force decrease. Second, lower stroke amplitude case present a more oscillated instantaneous force history in terms of root-mean-square value of force coefficient. A more quantitative data is shown in Table 1. 

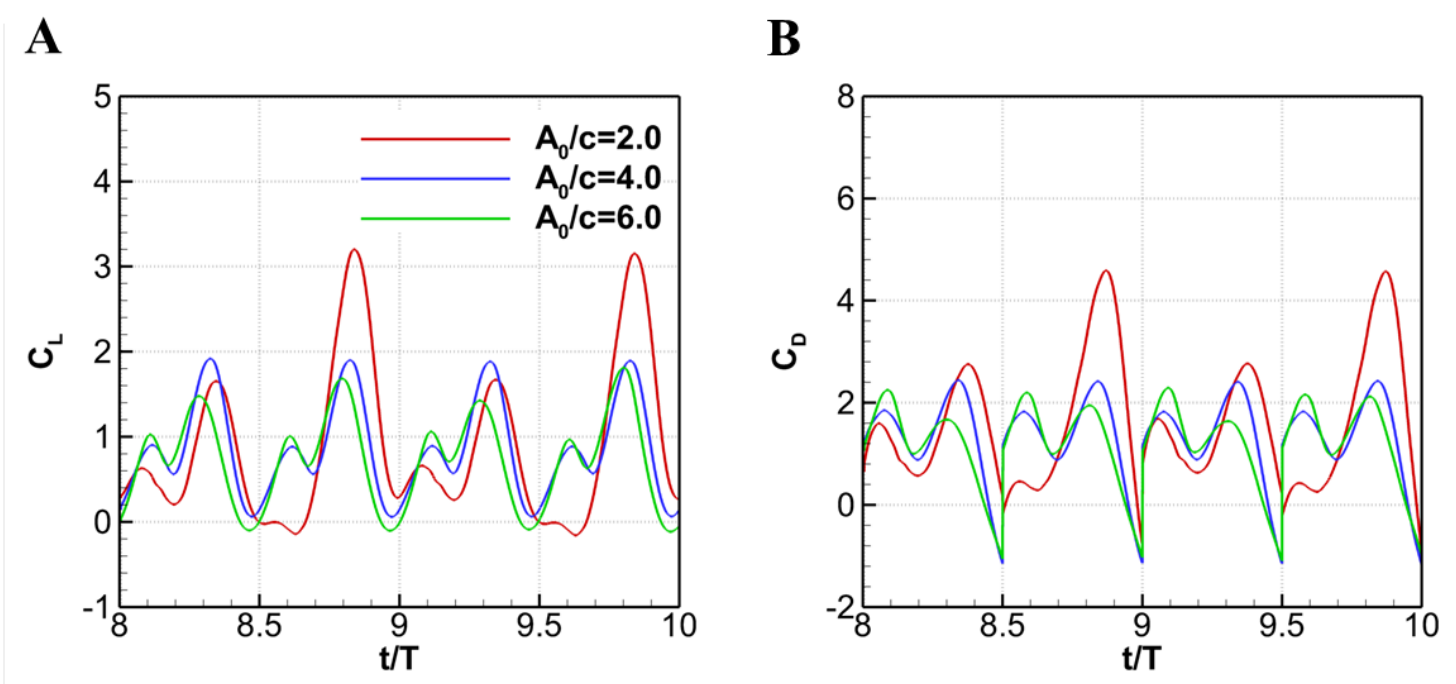

Figure 14: Stroke amplitude effects on lift coefficient (A) and drag coefficient (B) for rigid flat plates.

This actually is not a surprised result. By increasing the stroke amplitude, the reduced frequency $(k)$ was decreased, and thus the unsteadiness was suppressed. Observation from previous literature also proves this. A fractional factorial design method based study [25] numerically investigated more than ten parameters which will affect the trajectory of hovering kinematics. The authors concluded that the high flapping amplitude plays more important roles for the steadiness of aerodynamic response of hovering flapping wings. 
Table 1: Summary of lift and drag coefficient for flat flapping plates with different stroke amplitude

\begin{tabular}{ccccccc}
\hline \hline$A_{0} / c$ & $\overline{C_{L}}$ & $\left(C_{L}\right)_{\max }$ & $\left(C_{L}\right)_{r . m . s}$ & $\overline{C_{D}}$ & $\left(C_{D}\right)_{\max }$ & $\left(C_{D}\right)_{r . m . s}$ \\
\hline 2 & 0.89 & 3.19 & 1.34 & 1.61 & 4.59 & 2.11 \\
4 & 0.80 & 1.90 & 1.04 & 1.28 & 2.43 & 1.58 \\
6 & 0.73 & 1.67 & 0.92 & 1.19 & 2.19 & 1.47 \\
\hline \hline
\end{tabular}

Comparing to flat flapping plates, sounding flow will become more unsteady by adding a trailing-edge flap (TEF) dynamic motion. This actually equals providing additional energy to the near-wall fluid field and creating more disturbances to affect the leading-edge vortex (LEV) separation. It is also very interesting to know whether the unsteady aerodynamic performance will or will not benefit from this extra "unsteady source".

Figure 15 shows cycle-averaged force (lift and drag) coefficient and aerodynamic power coefficient time history against the trailing-edge deflection amplitude $\left(\beta_{T}\right)$. Each curve indicates a different value of stroke amplitude $\left(A_{0} / c\right)$. The first point of each curve presents the performance of a flat plate without TEF. For the rest series points, it shows the changing tendency of aerodynamic performance by increasing TEF deflection amplitude which is ranging from $10^{\circ}$ to $80^{\circ}$ respects to a symmetrical TEF rotation $\left(\varphi=0^{\circ}\right)$. In general, both dimensionless force and power coefficient increase with reduced frequency $(k)$. The difference between maximum and minimum values of each curve is smaller for larger stroke amplitude one. Furthermore, the drag force and 
aerodynamic power coefficient present similar trend with the increasing value of TEF deflection amplitude $\left(\beta_{T}\right)$, and gradually reach to peak values at $\beta_{T}$ around $60^{\circ}$. This is mainly because the drag force is the major component of power consumption for the horizontal stroke plane. As the deflection amplitude continually increasing, the drag force will reach to its limit since the effective projected area of deformed cannot unlimited varying. Unlike the monotonous changing tendency for drag and power coefficients, the lift coefficient curves show more diverse pattern. As shown in Figure 15(B), lower stroke amplitude cases present a bigger arch shape, such as lines ranging $2<A_{0} / c<4$. For higher ones, as the TEF deflection angle increasing, the lift coefficient decrease almost linearly. This indicates TEF actuation is more effective for smaller stroke amplitude comparing to larger ones. In other words, regardless the limited range of Reynolds number selected for the current investigation, the unsteady aerodynamic mechanism can benefit more from the chord-wise deformation for smaller stork amplitude flapping motion. If we use $75 \%$ span as the reference chord, the stroke amplitude angle for three-dimensional flapping is around $40^{\circ}$ to $90^{\circ}$ for aspect-ratio ranging from 2 to 6 . Beyond that, the stiffer wing might provide better aerodynamic performance. The results presented by Heathcote and Gursul [26,27] also showed that the deformation of flapping wing is not the more the better, but for modulating optimal vortex formation to generate more jet in vertical direction. 

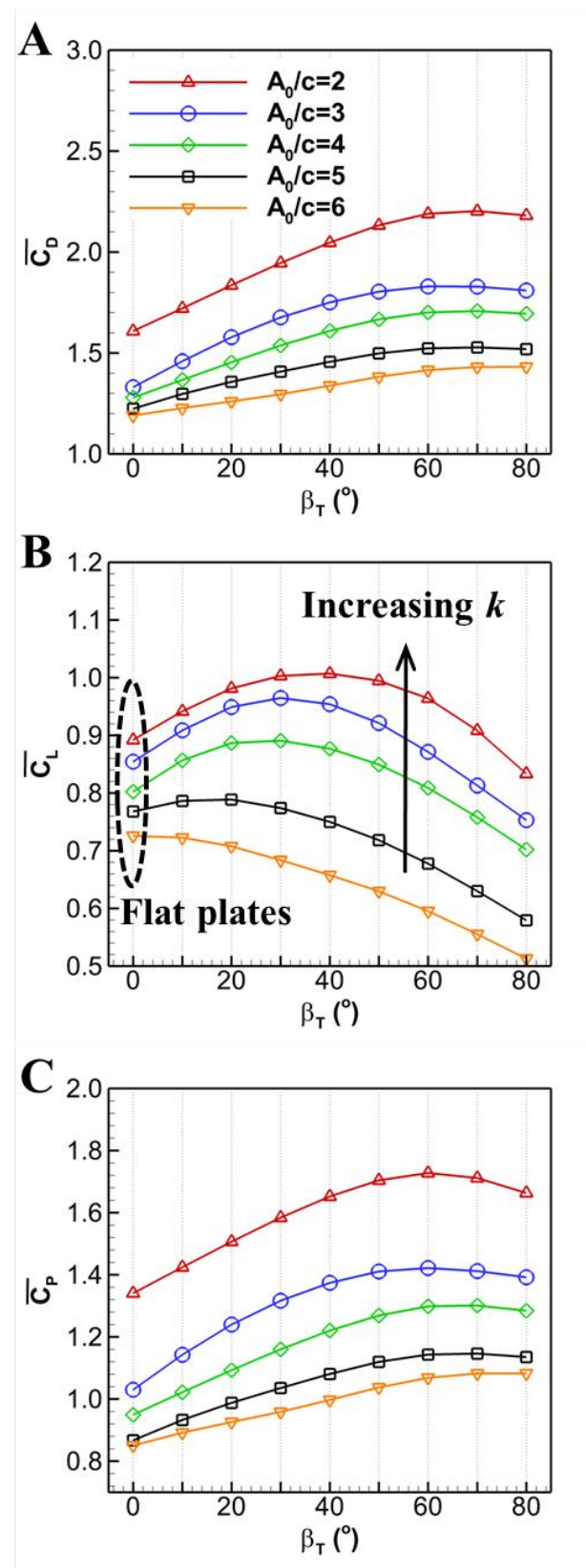

Figure 15: Cycle-averaged drag coefficient (A), lift coefficient (B), and aerodynamic power coefficient (C) plotted against the trailing-edge deflection amplitude $\left(\beta_{T}\right)$ for different flapping amplitude value $\left(A_{0} / c\right)$. In which, $\beta_{T}$ ranging from $10^{\circ}$ to $80^{\circ}$, and $\beta_{T}=0^{\circ}$ shows the performance of the rigid flat plate. In all above cases, the phase shaft $(\varphi)$ is zero and the Reynolds number was fixed at 100 . 
Except the force production and power consumption, aerodynamic efficiency is also another main concern for hovering. From Figure 16, we can see that in general the lift enhancement resulting from TEF comes at a cost of lower lift-to-drag ratio and aerodynamic efficiency in terms of lift-to-drag. Another thing we can learn from this plot is that although the lowest stroke amplitude $\left(A_{0} / c=2\right)$ cases present highest lift production, the aerodynamic efficiency is $30 \%$ lower than others, especially for smaller deflection amplitude. With the deflection amplitude increase, the efficiency for all cases is comparable.
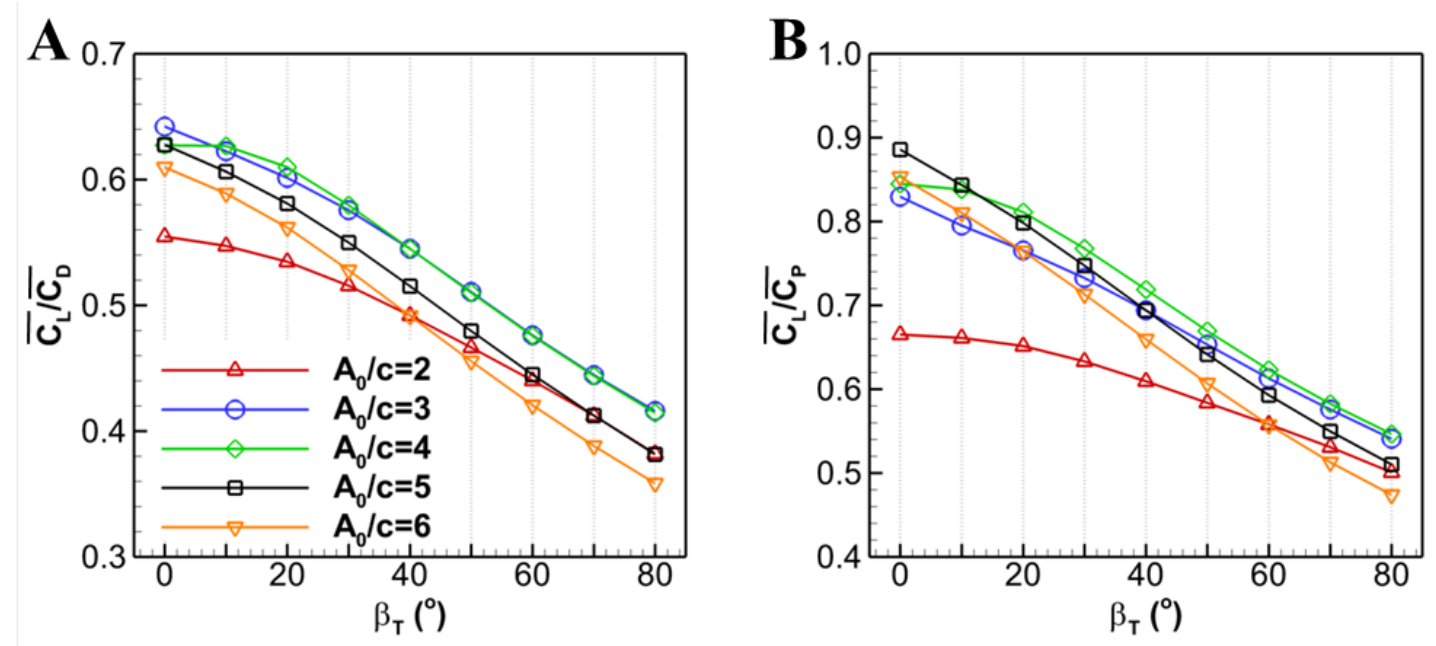

Figure 16: (A) Cycle-averaged lift-to-drag ratio; (B) Aerodynamic efficiency (lift-topower). In which, $\beta_{T}$ ranging from $10^{\circ}$ to $80^{\circ}$, and $\beta_{T}=0^{\circ}$ shows the performance of the rigid flat plate. In all above case the Reynolds number were fixed at 100.

Based on above study, we will select stroke amplitude $A_{0} / c=3$ for the continuing study. This selection has compromised on the lift production to gain higher aerodynamic efficiency. In the next subsection, a detailed parametric study will be presented to show 
how the trailing-edge flap deflection amplitude and phase shift affect the aerodynamic performance.

\subsection{Effects of Trailing-Edge Flap Deflection Amplitude and Phase Shift}

To explore the influence of trailing-edge flap kinematics on the aerodynamic performance, we calculated the mean force and power coefficients averaged throughout the stroke. They were evaluated for all pairs of trailing-edge deflection amplitude angle $\left(\beta_{T}=\left[10^{\circ}, 80^{\circ}\right]\right)$ and phase shift angle $\left(\varphi=\left[-80^{\circ}, 80^{\circ}\right]\right)$, at stroke amplitude fixed at $A_{0} / c=3$.

Figure 17 shows six sample simulations taken from the full set of 72 trials to illustrate how the magnitude and time course of aerodynamic forces vary with trailingedge deflection amplitude and phase shift. The force trajectories display prominent peaks at the middle of each half stork and minimum values during stoke reversal. Although the plate kinematics are symmetrical for all simulations, the force trajectories during the down-stroke and up-stroke do not similar at all because of complex unsteady effects. As numerous precious studies $[28,29,30]$ have shown that the aerodynamic performance result from an interaction of three distinct interactive mechanisms: delayed stall, rotational circulation, and wake capture. Since the major enhancement always appears on the peaks, it suggests that the translational mechanism effect is amplified by adding dynamic motion on trailing-edge flap. Another possibility is that the deformed plate might be present more effective angle of attack to catch the vortex shedding during previous stoke and benefit more from wake capture mechanism. To get better idea about 
how the force production was modified, more detailed information about the wake structure will be provided in the next section.
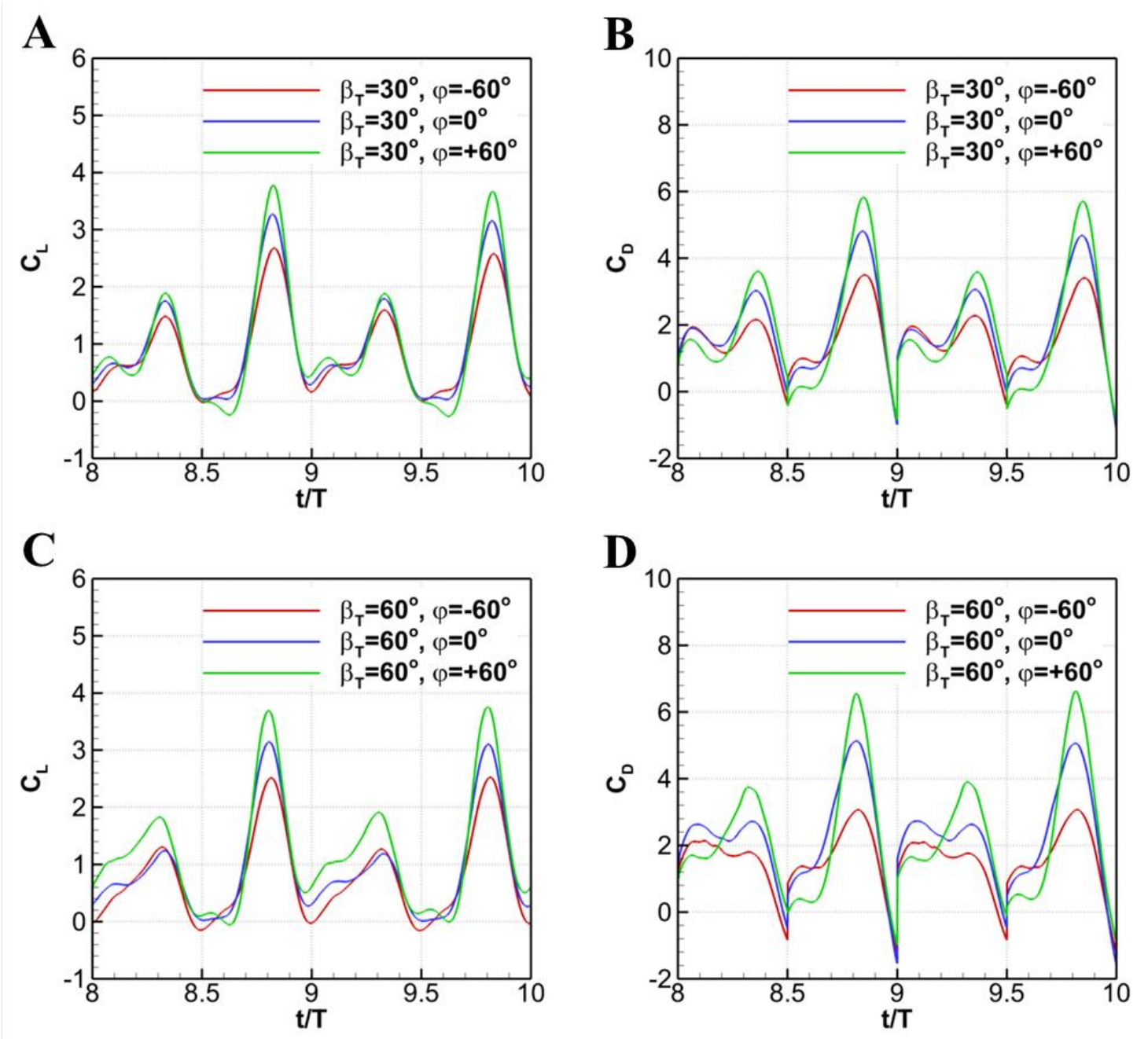

Figure 17: Sample instantaneous lift (A, C) and drag (B, D) coefficient for various combinations of trailing-edge deflection amplitude $\left(\beta_{T}\right)$ and phase shift $(\varphi)$. In each case, the flapping stroke amplitude is fixed at $A_{0} / c=3$, and Reynolds number is 100 .

To provide a more comprehensive picture of aerodynamic performances changing with different kinematics of trailing-edge flap (TEF), the maps for cycle-averaged lift 
coefficient, drag coefficient and aerodynamic coefficient are shown in Figure 18. The maps of lift-to-drag ratio and aerodynamic efficiency in terms of lift-to-power ratio are shown in Figure 19. In general, delayed TEF rotation $\left(\varphi=\left(0^{\circ}, 80^{\circ}\right]\right)$ exhibits a broad maximum range compared to advanced TEF rotation $\left(\varphi=\left[-80^{\circ}, 0^{\circ}\right)\right)$. For the map of mean lift coefficient $\overline{C_{L}}$, as shown in Figure 18(A), it is sensitive for the changing of both TEF deflection amplitude and phase shift. Moreover, there are optional configurations for $\beta_{T}$ and $\varphi$. The maximum $\overline{C_{L}}$ happens when $\beta_{T}=50^{\circ}$ and $\varphi=60^{\circ}$. As expected, map of mean drag coefficient $\left(\overline{C_{D}}\right)$ rise monotonically with increasing trailing-edge deflection amplitude $\left(\beta_{T}\right)$ for any given value of phase shift, and is not very sensitive to phase shift for advance TEF rotation range. Furthermore, the map of aerodynamic power coefficient (as shown in Figure 18C) is similar comparing to the drag coefficient.

It is interesting to perform the comparative study between flapping plates with and without trailing edge control, as shown in Figure 20. Flapping plate with such control could be aerodynamic force productive or energetic efficient over completely rigid plate. Table 2 shows the aerodynamic coefficients of cases with optimal configuration of trailing edge parameters and cases of completely rigid flapping plate. It is obvious that by selecting different combination of deflection amplitude and phase shift the cycleaveraged lift and efficiency enhancement can reach up to $27 \%$ and $14 \%$, respectively. 

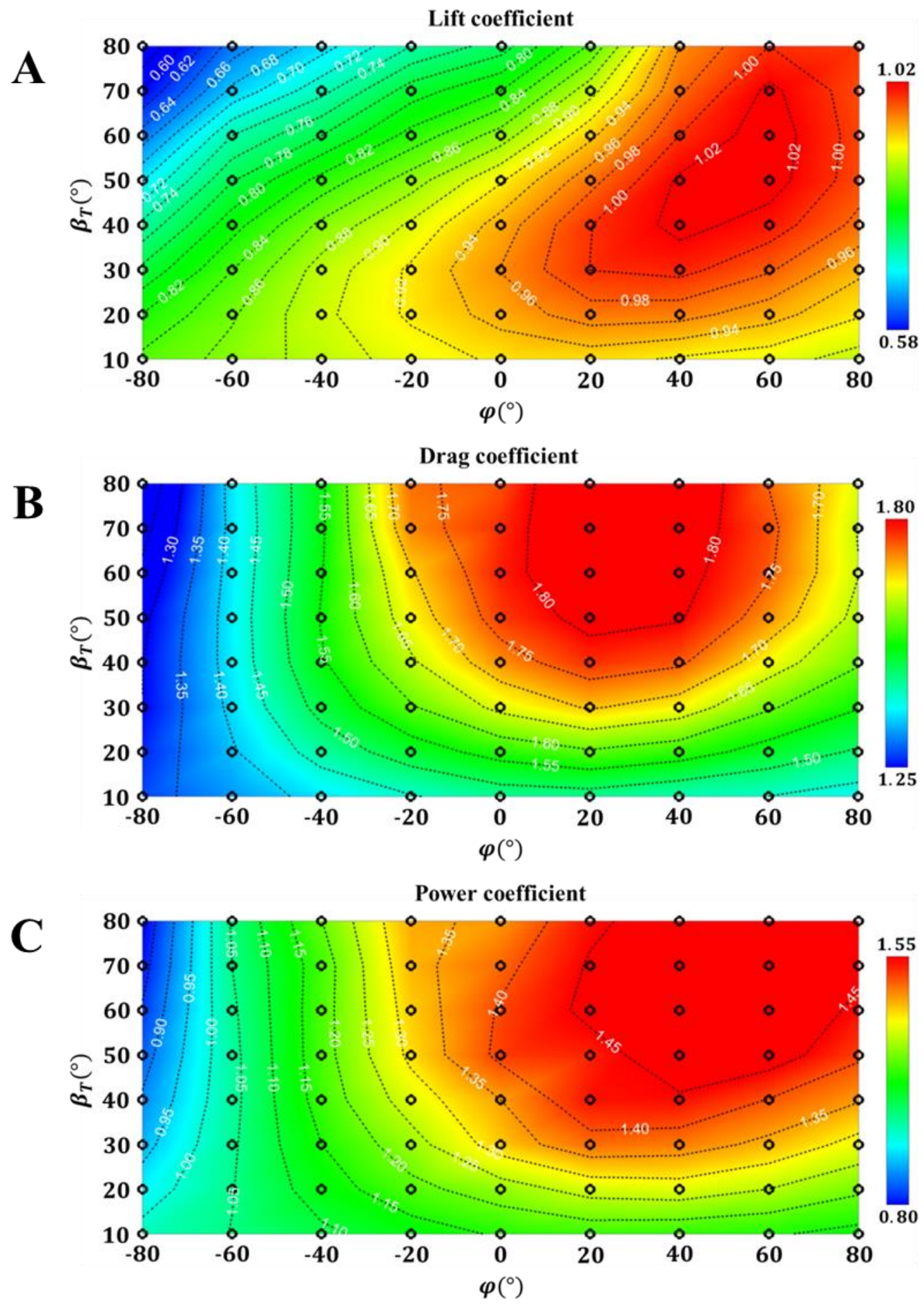

Figure 18: Parameter maps of cycle-averaged lift coefficient (A), drag coefficient (B), and aerodynamic power coefficient $(\mathrm{C})$ as function $\mathrm{s}$ of trailing-edge deflection amplitude and phase shift. 

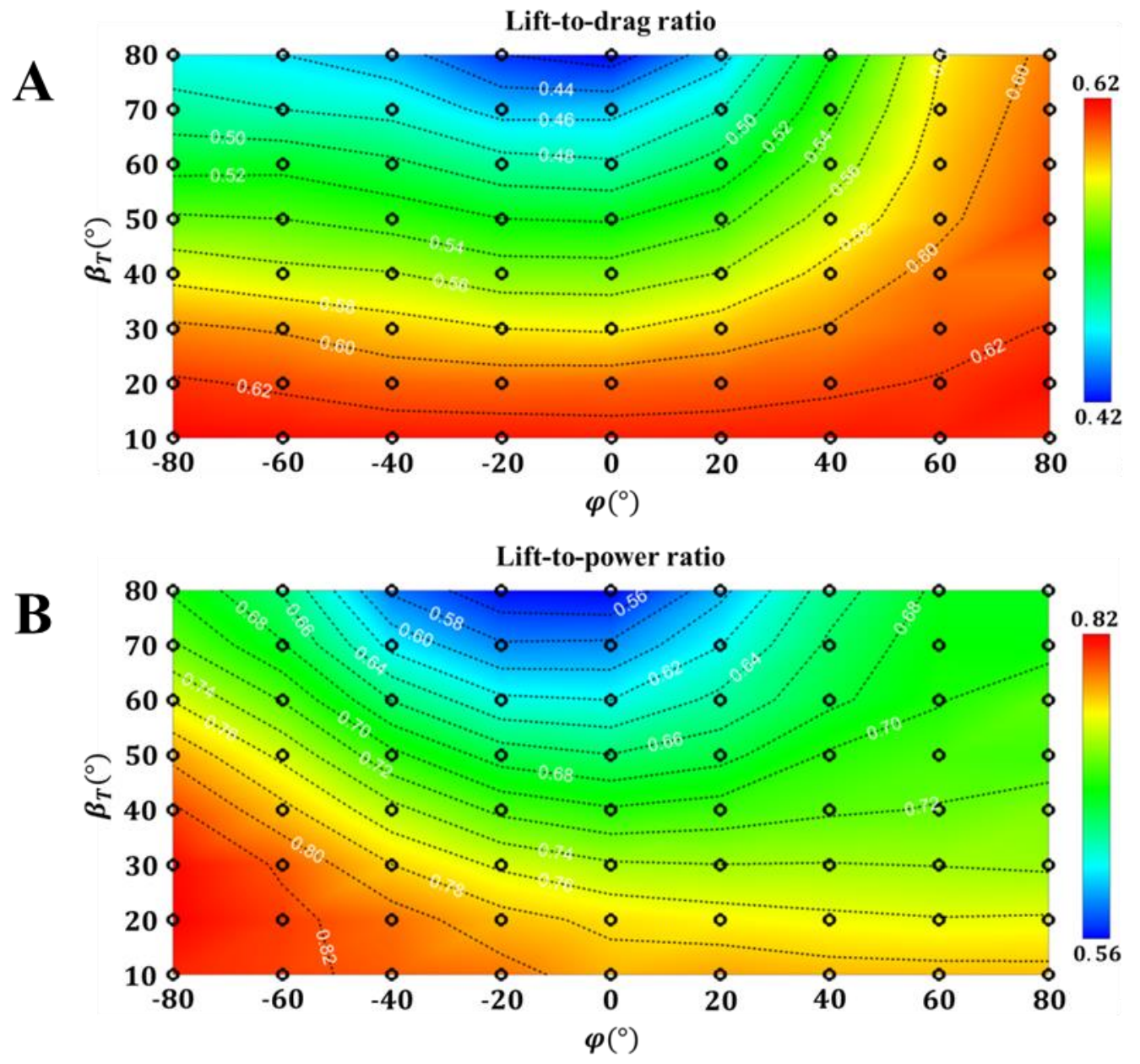

Figure 19: Parameter maps of cycle-averaged lift-to-drag ratio (A), and lift-to-power ratio (B) as function s of trailing-edge deflection amplitude and phase shift. 

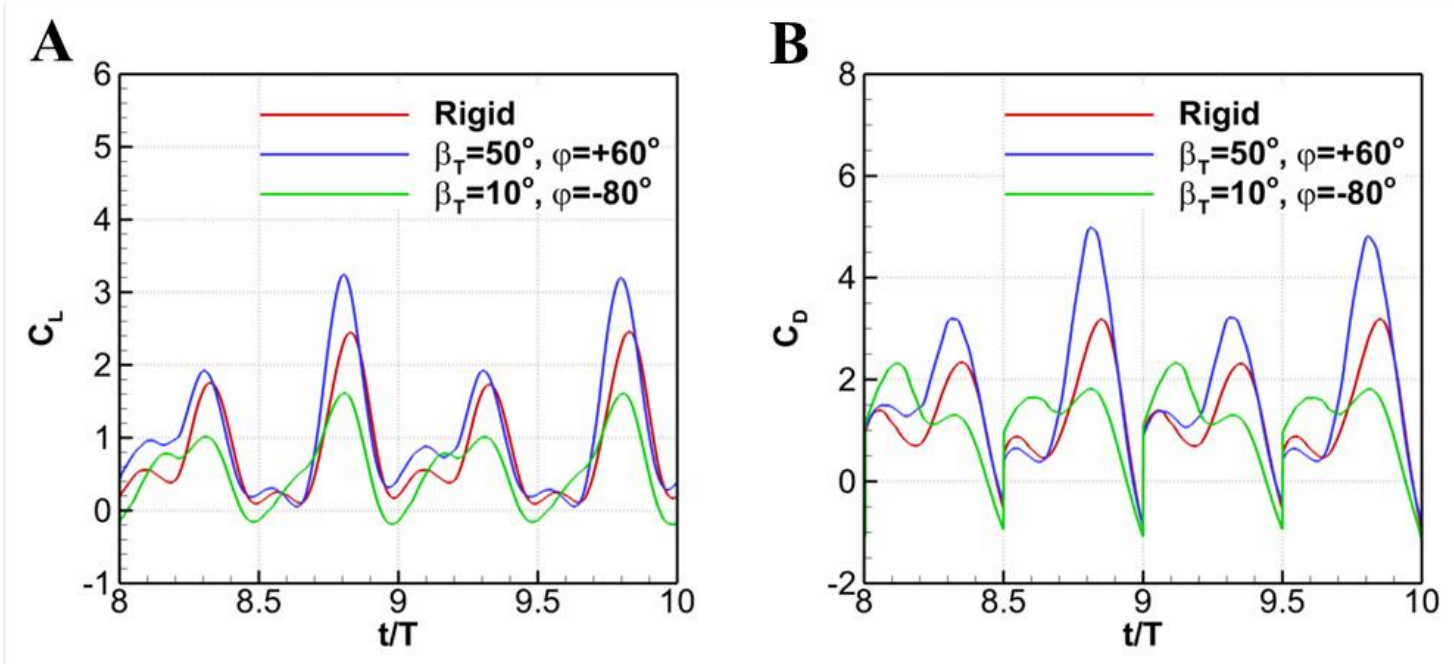

Figure 20: Instantaneous lift (A) and drag (B) coefficient for flapping plate without trailing-edge flap (Rigid), owning optimal cycle-averaged lift $\left(\beta_{T}=50^{\circ}, \varphi=+60^{\circ}\right)$, and owning optimal cycle-averaged aerodynamic efficiency $\left(\beta_{T}=10^{\circ}, \varphi=-80^{\circ}\right)$.each case, the flapping stroke amplitude is fixed at $A_{0} / c=3$, and Reynolds number is 100 .

Table 2: Comparative study of cases with and without TEF

\begin{tabular}{cccccccc}
\hline \hline Cases & $\beta_{T}$ & $\varphi$ & $\overline{C_{L}}$ & $\overline{C_{D}}$ & $\overline{C_{P}}$ & $\overline{C_{L}} / \overline{C_{D}}$ & $\overline{C_{L}} / \overline{C_{P}}$ \\
\hline 1.Rigid & $N / A$ & $N / A$ & 0.82 & 1.30 & 0.99 & 0.63 & 0.83 \\
2. Optimal & $50^{\circ}$ & $60^{\circ}$ & 1.04 & 1.75 & 1.46 & 0.59 & 0.71 \\
$\overline{C_{L}}$ & & & & & & & \\
3. Optimal & $10^{\circ}$ & $-80^{\circ}$ & 0.85 & 1.33 & 0.89 & 0.64 & 0.95 \\
$\overline{C_{L}} / \overline{C_{P}}$ & & & & & & & \\
\hline \hline
\end{tabular}




\subsection{Vortex Modulation Resulting from Trailing-Edge Flap}

In order to understand the lift enhancing mechanism through adding a dynamic trailing-edge flap (TEF), we further evaluate the vortex formation by comparing the optimal lift production case $\left(\beta_{T}=50^{\circ}, \varphi=60^{\circ}\right)$ to the rigid plate (without TEF). The instantaneous vorticity field in an entire flapping cycle and the corresponding plate configuration are shown in Figure 21. There are several similarities in the vortex behavior between the two cases. For instance, a leading edge vortex (LEV) is generated during each half-stroke and is then recaptured by the plate during its return trip after the stroke reversal (e.g. the positive vortex blob at $t / T=9.125$ and the negative blob at $t / T=9.625)$ The LEV moves downward along the plate and may merge with the trailing edge vortex (TEV) of the same sign that is being formed (e.g. the positive blob at $t / T=9.25$ and the negative blob at $t / T=9.75$ ). The merged vortex is strengthened and meanwhile stretched by the trailing edges as shown by the positive vortex band at $t / T=9.375$ and also by the negative band at $t / T=9.875$. The wake below the hovering plate is marked by a pair of TEVs with opposite signs that are generated by the two halfstrokes in a complete cycle.

The differences in the flow field between rigid plate and plate with trailing-edge flap $\left(\beta_{T}=50^{\circ}, \varphi=60^{\circ}\right)$ are also evident. First, the size of both LEV and TEV for flapping plate with trailing-edge flap is generally larger than the rigid one. Second, the TEVs pair below the hovering plate moves downward faster for plate with trailing-edge flap compering to the rigid one. This indicates that by adding a trailing-edge flap the induced velocity in vertical direction might be enhanced, and thus create a stronger jet like downwash in vertical direction. 


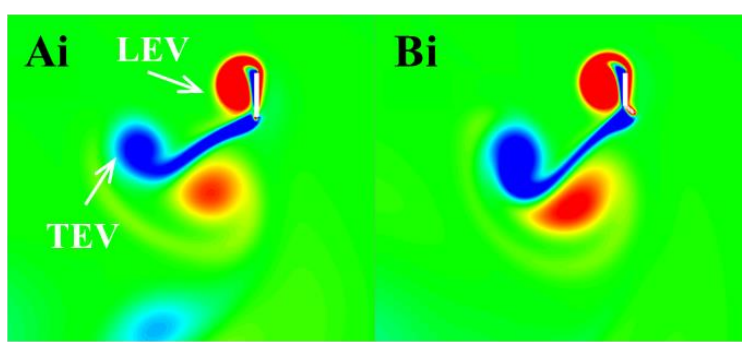

$\mathbf{t} / \mathbf{T}=\mathbf{9 . 0 0 0}$

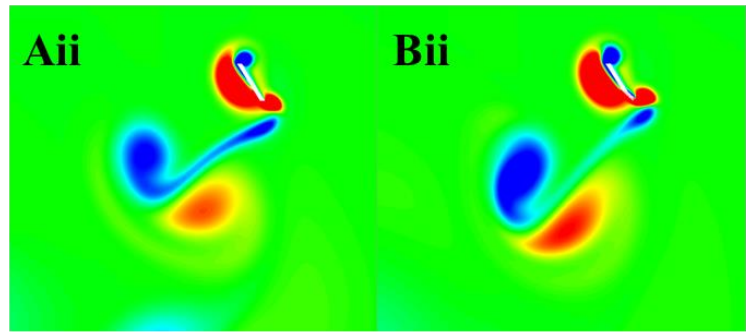

$\mathbf{t} / \mathbf{T}=\mathbf{9 . 1 2 5}$

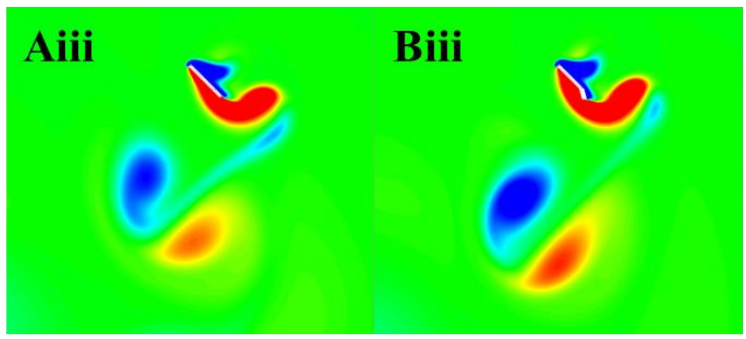

$\mathrm{t} / \mathrm{T}=\mathbf{9 . 2 5 0}$

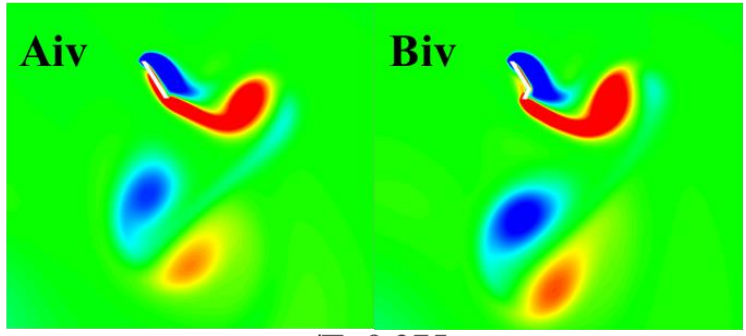

$\mathbf{t} / \mathbf{T}=\mathbf{9 . 3 7 5}$

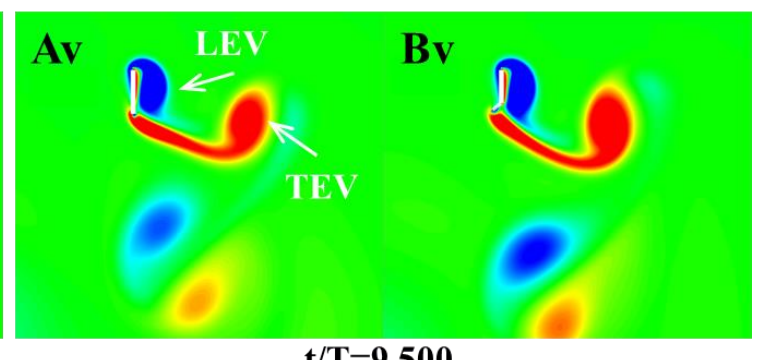

$\mathbf{t} / \mathbf{T}=\mathbf{9 . 5 0 0}$

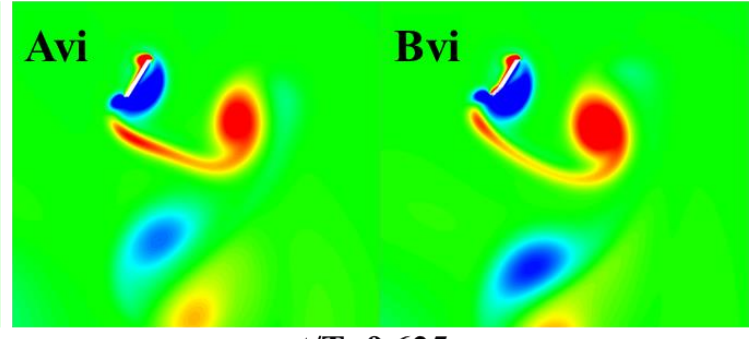

$\mathrm{t} / \mathrm{T}=\mathbf{9 . 6 2 5}$

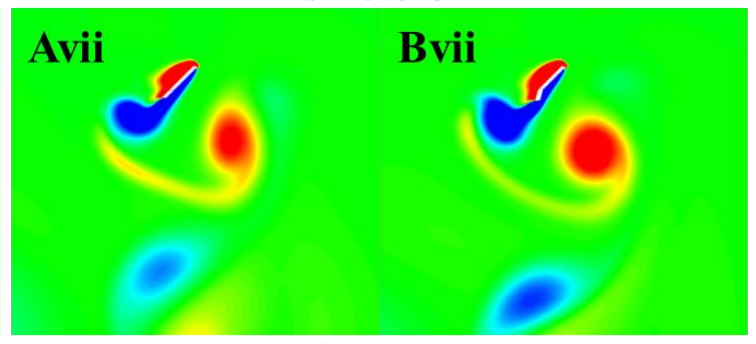

$\mathbf{t} / \mathbf{T}=\mathbf{9 . 7 5 0}$

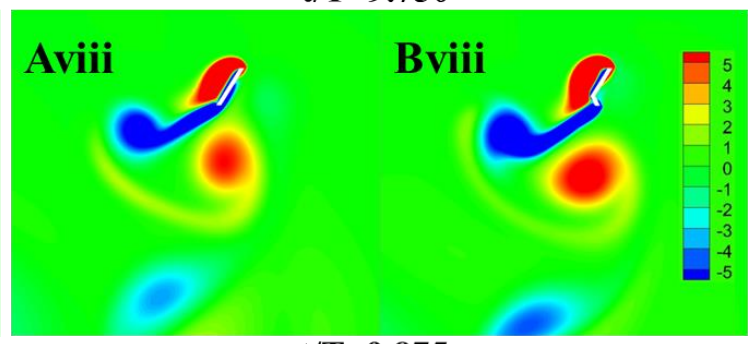

$\mathbf{t} / \mathbf{T}=\mathbf{9 . 8 7 5}$

Figure 21: A series of instantaneous vortex field in a flapping cycle for rigid plate (first and third columns) and plate with trialing-edge flap (second and fourth columns). The red and blue color indicates clockwise and anticlockwise, respectively.

The distinct vortices have been indicated in Figure 21. To quantify the strength of these vortices, I first visualized the vorticity field using contour lines. After each vortex is manually identified, a closed contour line is generated around this vortex with the 
specified level, and then the circulation $\Gamma$ is computation along this line. Thought the magnitude of the circulation depends on the chosen contour level, the characteristic behavior of the vortex is not affected by this choice. The computed circulation s of the LEV is shown in Figure 22 for the vortices indicated in Figure 21.

Generally speaking, the LEV circulation for optimal lift case $\left(\beta_{T}=50^{\circ}, \varphi=+60^{\circ}\right)$ is much stronger than the corresponding vortex for rigid one over a significantly long period in up-stroke. Comparing to up-stroke, down-stroke do not present much difference except during the plate start to reversal. The strength and timing of the LEV circulation have important consequences on the force production (Figure 20).

In order to understand this lift-enhancement mechanism of trailing-edge flap, the normalized pressure contours (shown in Figure 23) for both cases were compared during translational stage of down-stroke and up-stroke, respectively. During the translational stage, the LEV causes the flow to circulate around the leading-edge. The dynamic motion of trailing-edge flap feeds extra circulation to the TEV which induces a stronger downwash near the trailing edge. As a result, the vortex structure on the suction side is pulled down closer to the plate and backflow near the trailing edge is reduced. This leads to a larger range of low pressure field. Thus, the lift production is enhanced resulting from the increasing pressure difference between the upper and lower plate surface.

Figure 24 shows contours of the mean velocity in vertical direction where the mean is computed over the last two flapping cycles. Both plots show a high-intensity jet in the wake which is consistent with the fact that this is a lift-producing case. Also apparent is the asymmetry of this jet about the centerline. This asymmetry is consistent with the finding of $[31,32]$ where asymmetric wakes have been observed for a thrust-producing 
flapping foil. The direction of the wake deflection is determined by the starting motion of flapping plate. If the plate is started at the leftmost point and moved to right, a left deflection is obtained. However, once deflected towards one side of the centerline, the wake does not switch sides in a given simulation. The asymmetry produced a mean fore in horizontal direction that is significantly lower in magnitude than the mean lift force and therefore has little dynamical significance.

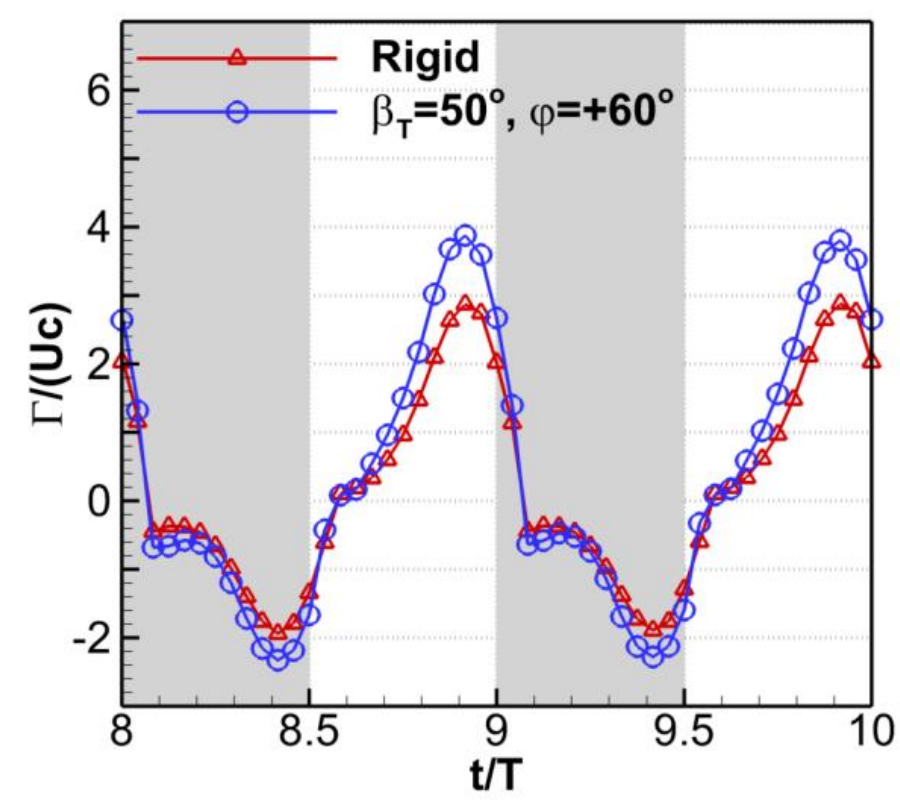

Figure 22: Comparison of leading-edge vortex (LEV) circulation between rigid plate without trailing-edge flap (TEF) and with TEF dynamic motion $\left(\beta_{T}=50^{\circ}, \varphi=60^{\circ}\right)$. The LEV circulation is nondimensionalized using reference velocity $(U)$ and chord length $(c)$. The gray shades indicate the down-stroke periods. The positive and negative values correspond to the strength of LEV during down-stroke and up-stroke, respectively. 


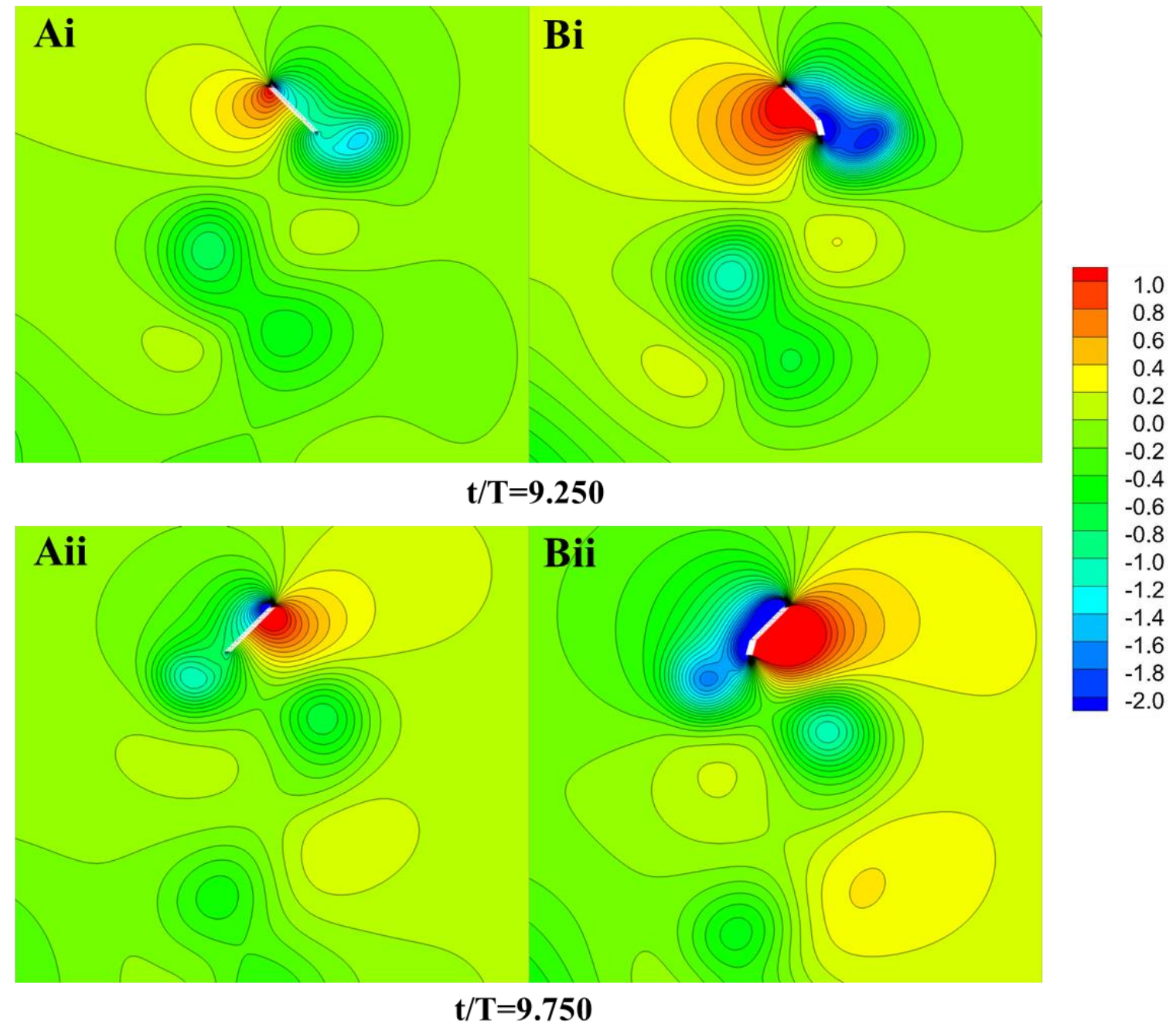

Figure 23: Pressure contour comparison between rigid plate (A) and plate hinged with trailing-edge flap (B). The pressure is normalized by using reference velocity.

The differences in the mean velocity contours (Figure 24) between rigid plate and plate with trailing-edge flap $\left(\beta_{T}=50^{\circ}, \varphi=60^{\circ}\right)$ are also evident. The high-intensity jet is much stronger for the plate with trailing-edge flap. This provides another evidence to show the lift production is enhanced by creating stronger downwash. Also the deflection of the asymmetry jet also increased. 

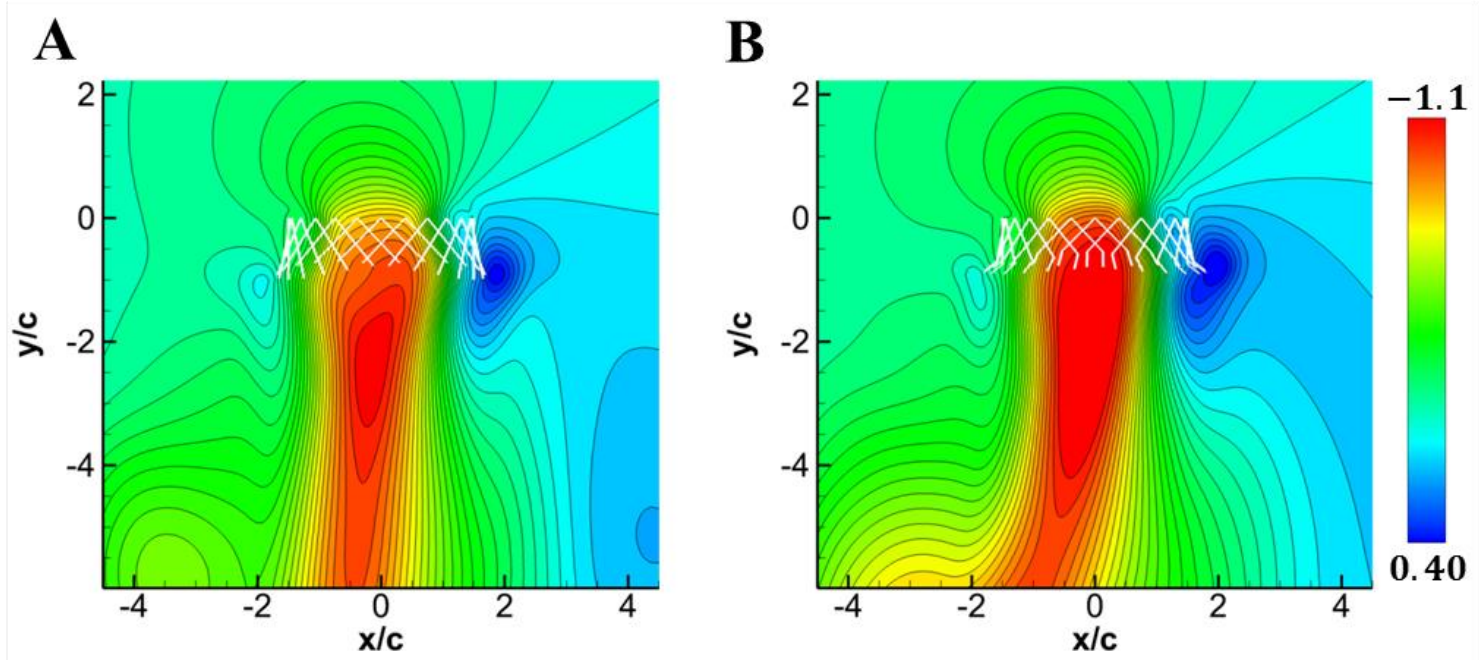

Figure 24: Contour of mean velocity (in vertical direction) corresponding to plate flapping configurations. (A) Rigid plate without trailing-edge flap; (B) Plate with trailingedge flap dynamic motion at $\beta_{T}=50^{\circ}, \varphi=60^{\circ}$. 


\section{Chapter 4: Three-Dimensional Hovering Motion with Trailing-}

\section{Edge Flap}

Unsteady aerodynamic performance of deformed flapping plated has been considered for two-dimensional flows in last chapter. In this chapter, the performance exerted upon the plate with the three-dimensional wake vortices will be considered. Three-dimensional effect such as aspect-ratio and geometry of trailing-edge flap will be studied.

\subsection{Plate Geometry and Kinematics}

The geometry considered in this study is a rectangular plate of varying aspect ratio with zero thickness membrane. The root of the plate is extended out a distance of $r_{\text {root }}=0.5 c$ from the axis of rotation. The plate follows a sinusoidal sweeping and pitching motion in an idealized horizontal stroke plane. Since there is no deflection on the stroke plane, the trajectory of a rigid plate can be described by two degrees of freedom. One is the leading-edge position angle ( $\Psi$ ) in spherical coordinates, and another is the pitching angle $(\Theta)$ about the leading-edge. The plate motion is thus specified by two periodic functions: $\Psi(t)$ and $\Theta(t)$. It is impractical to enumerate this family of kinematics by brute-force approach. The model chosen for this chapter is one of the simplest possible families of a hovering motion but it allows studying the dependence of force and flow by applying trailing-edge dynamic motion.

In particular, a three-dimensional plate flapping plate follow the motion governing by Eq. (15) and (16). The kinematics configuration shows in Figure 25. 


$$
\begin{gathered}
\Psi(t)=\Psi_{m} \cos \left(2 \pi f t+\phi_{\Psi}\right) \\
\Theta(t)=\Theta_{m} \sin \left(2 \pi f t+\phi_{\Theta}\right)+\Theta_{0}
\end{gathered}
$$

Where, $\Psi(t)$ and $\Theta(t)$ denote the rotational motion in azimuthal coordinate and pitching coordinate, respectively, $f$ is the flapping frequency, $\Psi_{m}$ and $\Theta_{m}$ are the amplitudes of sweeping and pitching, respectively, $\phi_{\Psi}$ and $\phi_{\Theta}$ are the phase offset of sweeping and pitching, respectively, $\Theta_{0}$ is the initial pitching angle.

A

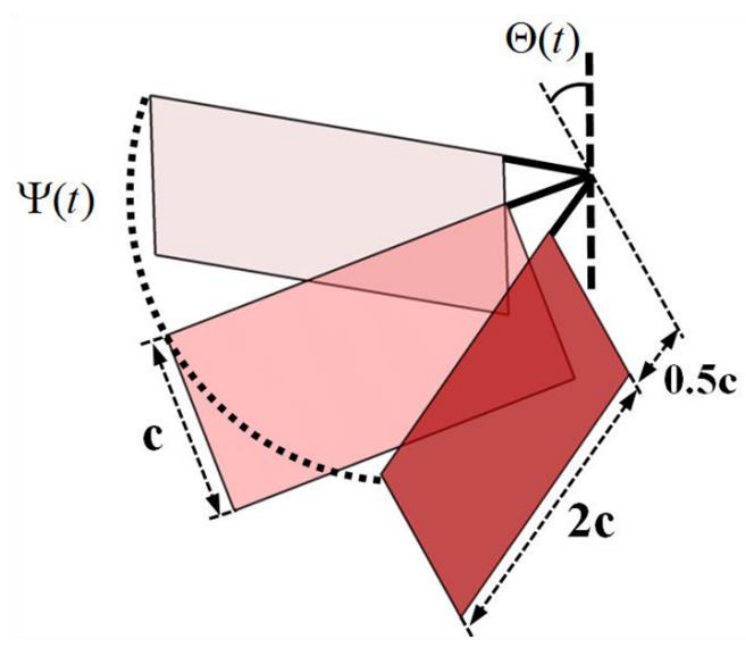

B

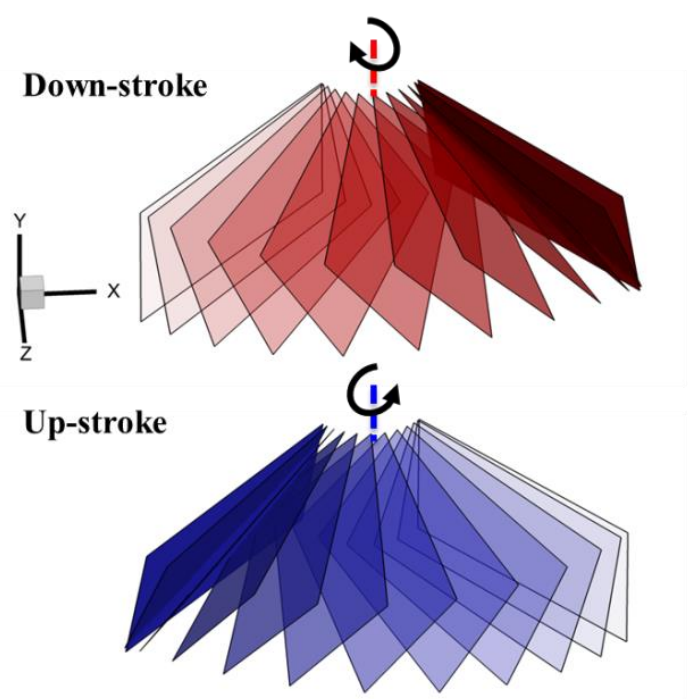

Figure 25: Three-dimensional flapping plate kinematics. (A): Plate geometry and definition of kinematics parameters; (B): Flapping plate configuration during downstroke (red) and up-stroke (blue).

For study the effect of aerodynamic function of trailing-edge flap. A trailing-edge flap, accounting for $25 \%$ of the total area of plate, is hinged on the plate with three different shapes, as shown in Figure 26. The same as previous two-dimensional study, the 
deflection angle $\left(\theta_{T}\right)$ of trailing-edge flap is prescribed by Eq. (7) with respect to the hinge axis for mimicking time-varying camber deformation in three-dimensional space.
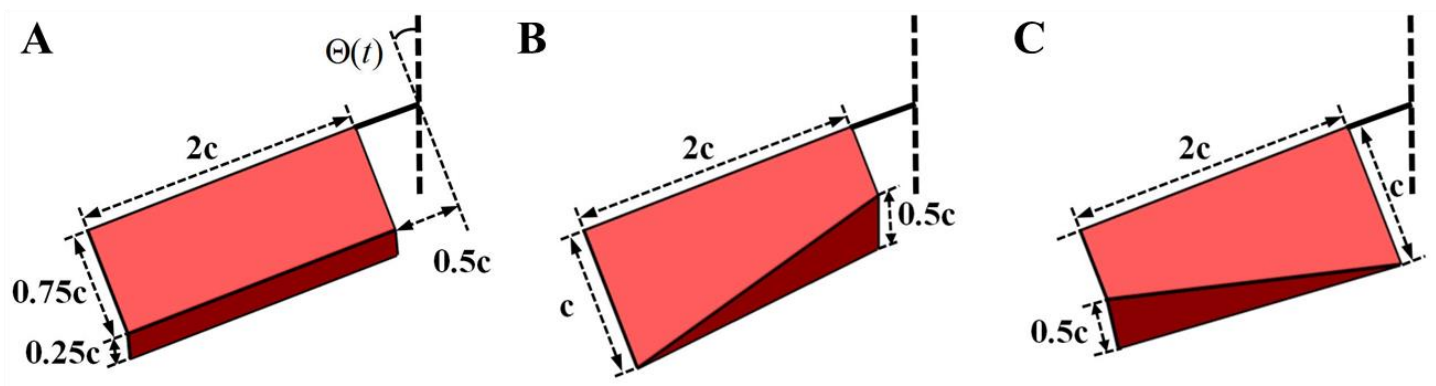

Figure 26: Three types of trailing-edge shape. (A) Rectangular shape (TS); (B) Left triangle shape (LTS); (C) Right triangle shape (RTS).

\subsection{Normalization and Choice of Parameters}

Similar as previous two-dimensional study, the three-dimensional flapping motion of the plate is completely specified by two dimensionless parameters, the Reynolds number ( $R e$ ), and Reduced frequency ( $k$ ), given by Eq. (17) and (18):

$$
\begin{gathered}
R e=\frac{U_{r e f} c}{v}=\frac{\pi f \Psi_{m} R_{75} c}{v} \\
k=\frac{\pi f c}{U_{r e f}}=\frac{c}{\Psi_{m} R_{75}}
\end{gathered}
$$

Where, $U_{r e f}$ is the reference velocity, $R_{75}$ is the representative radius which chosen as $75 \%$ of the tip radius, and $c$ is the chord length. In current study, the maximum plate velocity of leading-edge is used as the reference velocity, written as $U_{\text {ref }}=\pi f \Psi_{m} R_{75}$.

The Reduced frequency $(k)$ provides a measure of unsteadiness associated with a flapping wing via spatial wavelength of the flow disturbance with the chord length. Note 
that the reduced frequency is inversely proportional to the product of plate azimuthal angle amplitude and reference rotational radius, but not related to flapping frequency.

Similar as two-dimensional definition, in current three-dimensional study, the performance of the flapping plates is to be measured in terms of force (vertical and horizontal) generation and power expenditure. The classical way of assessing and comparing these quantities is through non-dimensionalized coefficients. This nondimensionalization also requires one to choose an appropriate velocity scales as the aforementioned $U_{\text {ref }}$. Then, the force coefficients can be defined as:

$$
\begin{aligned}
C_{L} & =\frac{2 F_{L}}{\rho U_{r e f}^{2} S} \\
C_{D} & =\frac{2 F_{D}}{\rho U_{r e f}^{2} S}
\end{aligned}
$$

Where, $F_{L}$ and $F_{D}$ are the vertical (lift) and horizontal (drag) force, respectively, $S$ is the plate surface area, and $C_{L} C_{D}$ are the corresponding coefficients.

The instantaneous aerodynamic power was calculated as $P=\sum_{i=1}^{n} \overrightarrow{F_{i}} \cdot \overrightarrow{v_{i}}$, where $\mathrm{n}$ is total number of triangular element on the wing, $\vec{F}_{i}$ is the aerodynamic force on each element and $\overrightarrow{v_{i}}$ is the corresponding velocity of the element. Following a similar approach, the power coefficient can be expressed as:

$$
C_{P}=\frac{2 P}{\rho U_{r e f}^{3} S}
$$

Where, $C_{P}$ is the power coefficient and $P$ is the aerodynamic power expended by the plates. 
The cycle-averaged aerodynamic efficiency (lift-to-power ratio) is used as a metric of aerodynamic performance in accordance with the ratio of lift-to-drag ratio, which is defined as:

$$
\eta_{\text {aero }}=\overline{C_{L}} / \overline{C_{P}}
$$

\subsection{Case Setup and Grid Independent Study}

Figure 27(A) shows the flapping plate configuration immersed in the threedimensional non-uniform Cartesian grid. The coordinate directions, which are also shown in Figure 27(B), are as follows: $\mathrm{X}$ is in the horizontal direction with $+\mathrm{X}$ pointing towards the up-stork direction; $\mathrm{Z}$ is the span-wise direction with $+\mathrm{Z}$ pointing to the tip of the plate; and $\mathrm{Y}$ is the vertical direction with $+\mathrm{Y}$ pointing upward.
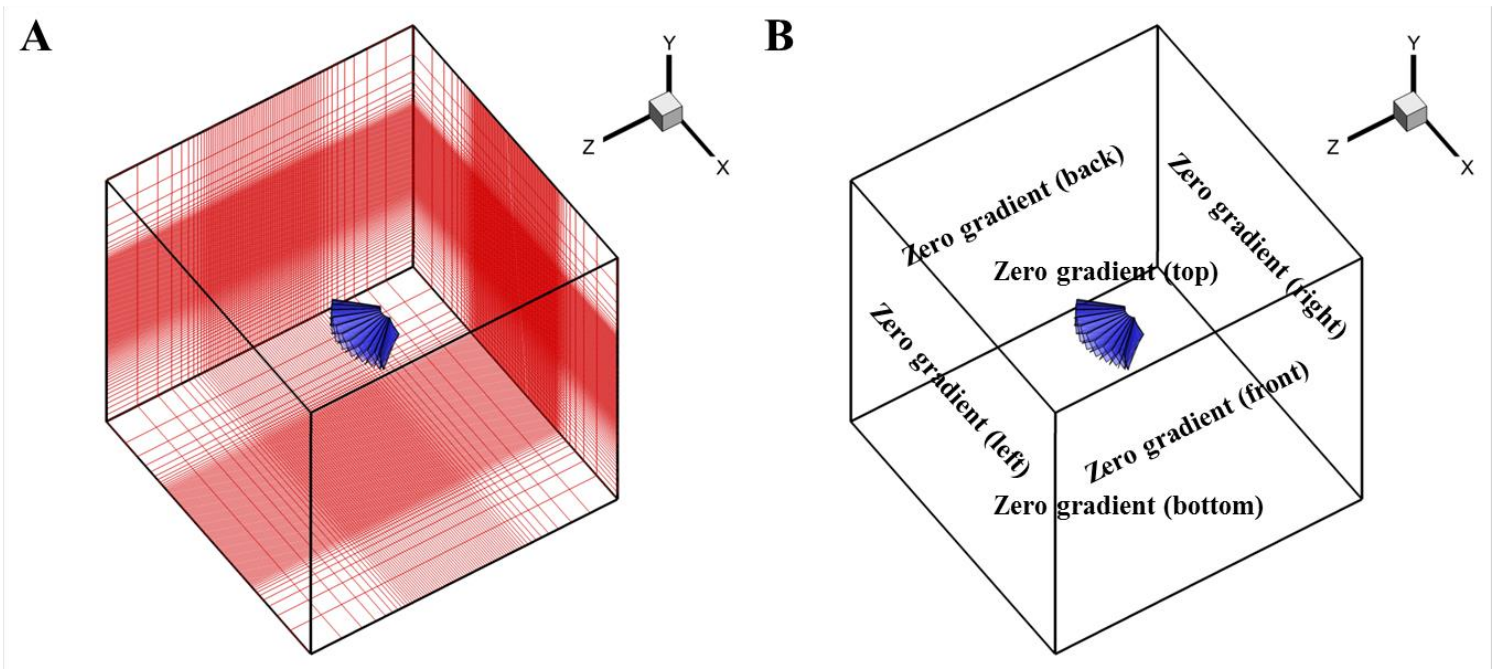

Figure 27: (A) Three-dimensional flapping plate configuration immersed in the twodimensional non-uniform Cartesian grid; (B) Boundary conditions for the simulation. 
Figure 27(B) shows the boundary conditions applied on the computational domain boundaries. On all of the boundaries, a far-field boundary condition which amounts to specifying the stream-wise (vertical) component to zero and setting the normal gradients of the other velocity components to zero is applied. The final domain size normalized by the chord length $(c)$ is $30 \times 30 \times 30$ and this large domain ensures that the boundaries do not have any significant effect on the computed results. As shown in Figure 27(A) the grid is designed to provide high resolution in the region around body as well as the wake which is expected to develop below the plate configuration. The final grid adopted here has $137 \times 161 \times 77$ to $233 \times 161 \times 161$ non-uniform mesh for different aspect-ratio case with the smallest resolution of $\Delta x=\Delta y=0.02$. This corresponds to 50 grids across the chord -wise direction on plate surface.

Three additional simulations on different grids were carried out to assess the convergence of the computed flow. Simulation 1 was carried out on a grid which had $70 \%$ more grid points than the baseline grid and simulation 2 was carried out on a mesh with $50 \%$ less grid points than the baseline grid. Both of these simulation s produced a maximum $2 \%$ difference from the baseline in mean lift and root-mean-squres (r.m.s) values of lit and drag. Finally simulation was carried out on a grid where the number of grid points in each direction around the plate was twice those in simulation 2. This simulation also produce mean lift and r.m.s lift and drag that are with $2 \%$ of simulation 2 and take together with other simulations, provide clear proof of the grid convergence of the current results.

The time-step chose for the current simulation is $\sim 2.08 \times 10^{-3}$ which corresponds to a Courant-Friedrichs-Lewy (CFL) number of $\sim 0.61$. With this time step, each flapping 
cycle takes 480 time-steps. In the current work, each simulation was integrated over four flapping cycles. Estimates of cycle mean and r.m.s lift as well as r.m.s drag that there is a less than a $1 \%$ difference in these quantities from the third to the fourth cycle. Thus, the flow quantities reach a near-stationary state by the third cycle and all of the flow statistics in the following discussion are estimated based on averaging over the third and fourth cycles.

\subsection{Effects of Aspect-Ratio}

Figure 28 gives the time courses comparison of lift coefficient $\left(C_{L}\right)$ and drag coefficient $\left(C_{D}\right)$ of plates with $A R=1,2$ and 4 in a completed third and fourth flapping cycles for a fixed Reynolds number 100. The instantaneous aerodynamic performance generated on the plate for different aspect ratio cases share the similar tendency for both lift and drag coefficient. Overall, in the current flow condition, $A R=1$ case owns relative better performance in terms of lift production. Moreover, $A R=4$ case owns relative better efficiency in terms of lift-to-power ratio. The cycle-averaged force production and power consumption shows in Table 3.

Table 3: Comparison of mean lift coefficient $\left(\overline{C_{L}}\right)$, drag coefficient $\left(\overline{C_{D}}\right)$, aerodynamic power coefficient $\left(\overline{C_{P}}\right)$, lift-to-drag ratio $\overline{C_{L}} / \overline{C_{D}}$, and lift-to-power ratio $\overline{C_{L}} / \overline{C_{P}}$ for different aspect-ratio

\begin{tabular}{cccccc}
\hline \hline$A R$ & $\overline{C_{L}}$ & $\overline{C_{D}}$ & $\overline{C_{P}}$ & $\overline{C_{L}} / \overline{C_{D}}$ & $\overline{C_{L}} / \overline{C_{P}}$ \\
\hline 1.0 & 0.652 & 1.222 & 1.097 & 0.53 & 0.59 \\
2.0 & 0.644 & 0.986 & 0.782 & 0.65 & 0.82
\end{tabular}


A

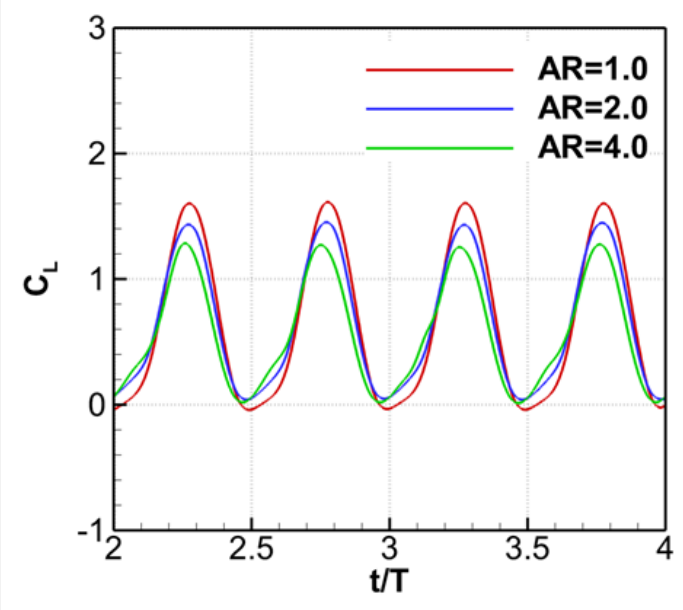

B

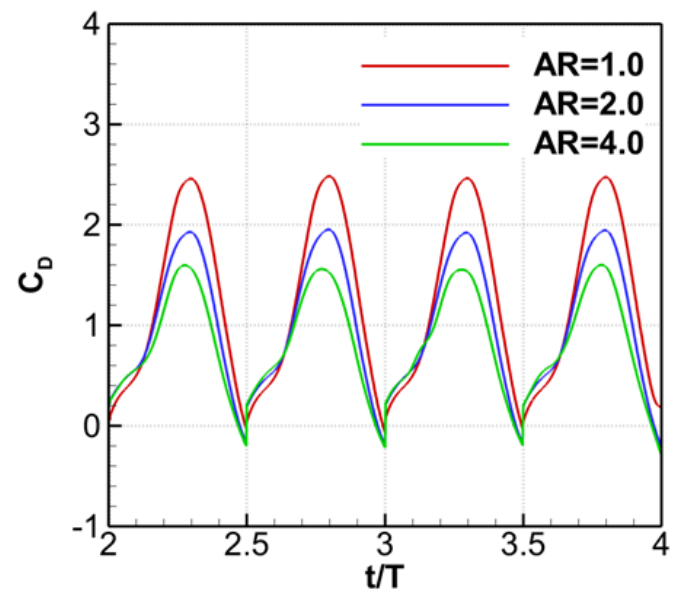

Figure 28: Comparison of instantaneous lift coefficient (A) and drag coefficient (B) for different aspect-ratio ( $A R=1,2,4)$ during the third and fourth flapping cycle.

Figure 29 shows the surface pressure distribution on the plate top surface at the middle of down-stroke corresponds to instants of the maximum force difference period. During the flapping motion, the significant low pressure area is located close to the leading edge near the tip due to the attached leading-edge vortex (LEV). For the case with best efficiency case, $A R=4$, there exist a relative large low pressure area at the corner of tip. On the contrary, a larger lower pressure area present close to the tip area for smallest aspect-ratio case $A R=1$. 


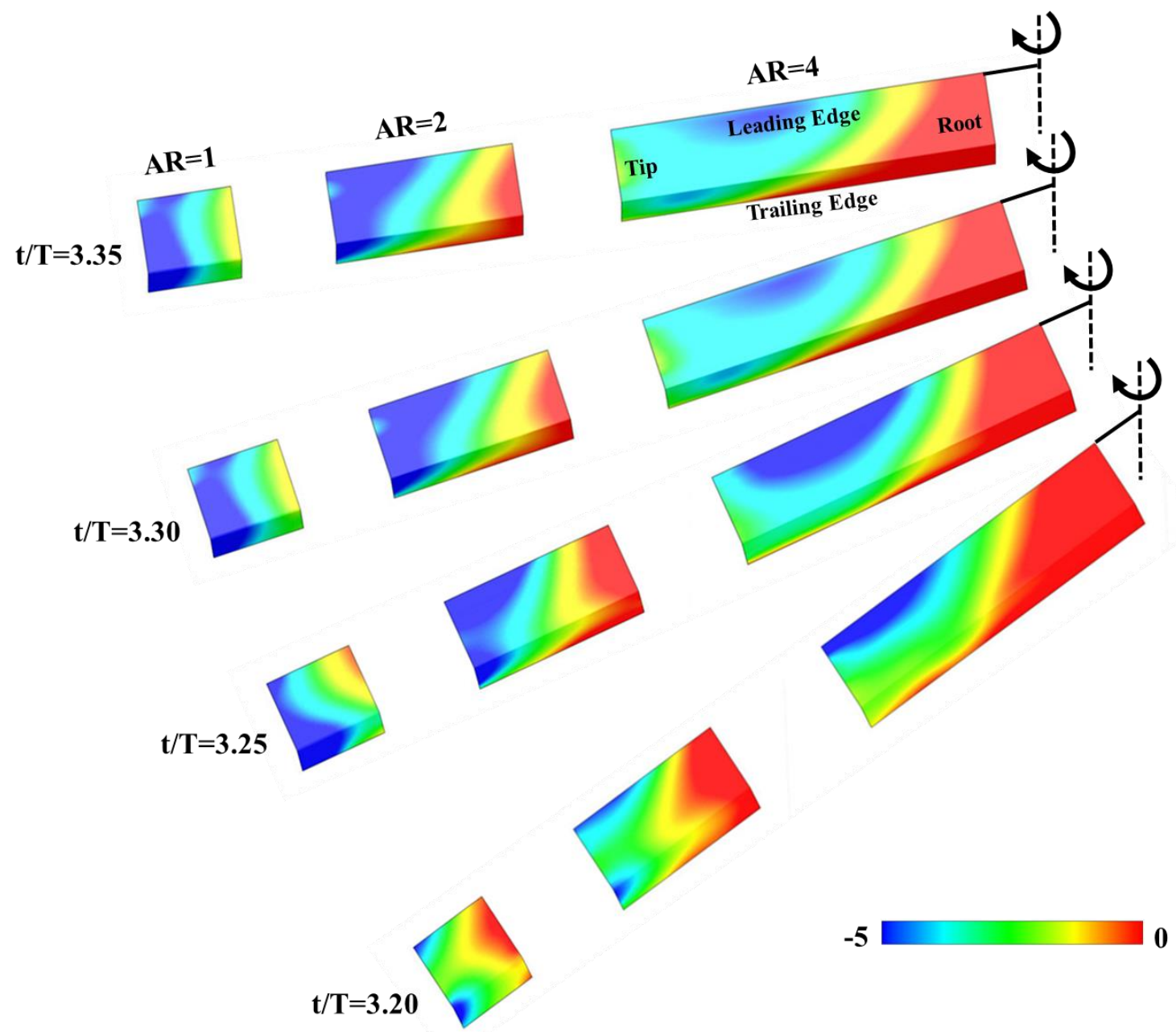

Figure 29: Evolution of the surface pressure distribution on the suction side of the plate with different aspect-ratio. The pressure is normalized by the reference velocity.

\subsection{Effects of Trailing Edge Shape}

Figure 30 gives the time courses comparison of lift coefficient $\left(C_{L}\right)$ and drag coefficient $\left(C_{D}\right)$ of plates with different trailing-edge flap (TEF) shape in a completed third and fourth flapping cycles for a fixed Reynolds number 100. The instantaneous 
aerodynamic performance generated on the plate for different TEF shape cases share the similar tendency for drag coefficient but not for lift coefficient. Overall, in the current flow condition, both RS and RTS cases own relative better performance in terms of lift production. The cycle-averaged force production and power consumption shows in Table 4.

Figure 31 presents the comparison of surface pressure distribution on the suction surface of plate with different trailing-edge flap shape. By adding the trailing-edge flap, the whole surface pressure distribution was changed tremendous. This change also results in a flow filed modification which can be seen from Figure 31(B).
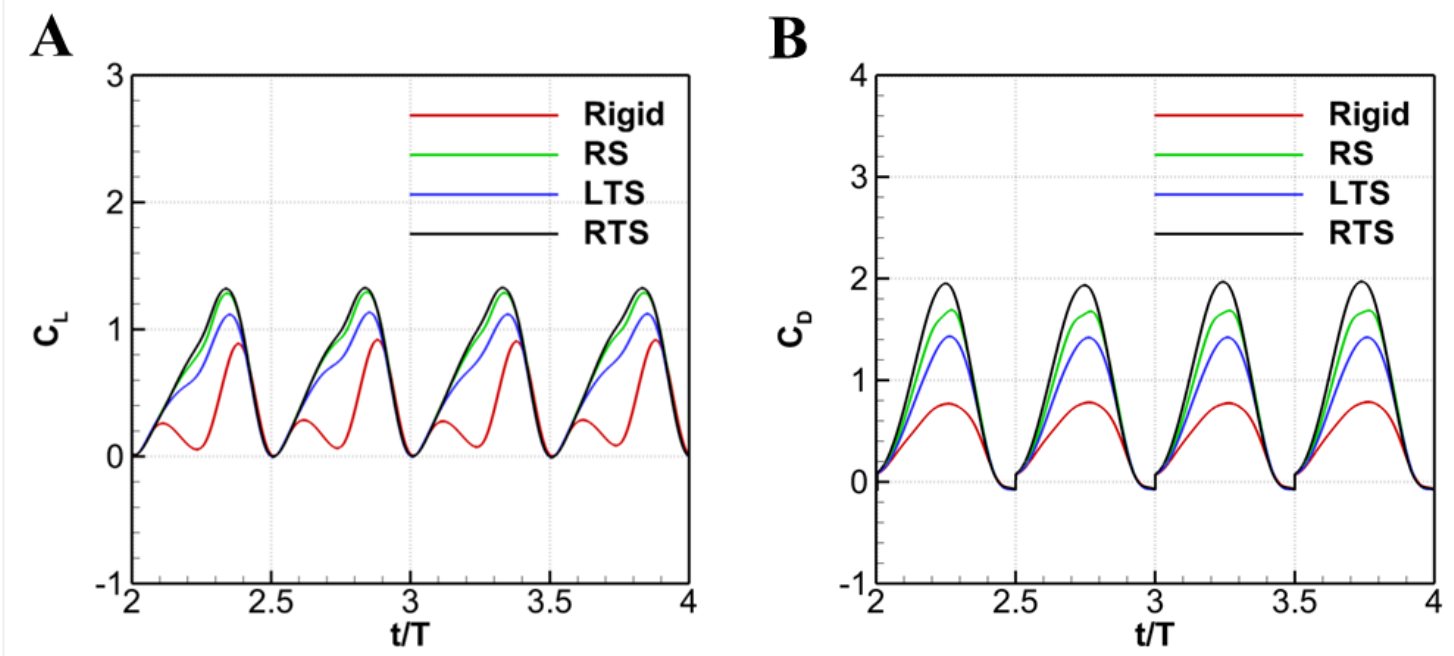

Figure 30: Comparison of instantaneous lift coefficient (A) and drag coefficient (B) for different trailing-edge shape (Rigid: without trailing-edge flap; RS: rectangular trailingedge shape; LTS: left triangular trailing-edge shape: RTS: right triangular trailing-edge shape) during the third and fourth flapping cycle. 
Table 4: Comparison of mean lift coefficient $\left(\overline{C_{L}}\right)$, drag coefficient $\left(\overline{C_{D}}\right)$, aerodynamic power coefficient $\left(\overline{C_{P}}\right)$, lift-to-drag ratio $\overline{C_{L}} / \overline{C_{D}}$, and lift-to-power ratio $\overline{C_{L}} / \overline{C_{P}}$ for different trailing-edge flap (TEF) shape.

\begin{tabular}{cccccc}
\hline \hline TEF Shape & $\overline{C_{L}}$ & $\overline{C_{D}}$ & $\overline{C_{P}}$ & $\overline{C_{L}} / \overline{C_{D}}$ & $\overline{C_{L}} / \overline{C_{P}}$ \\
\hline Rigid & 0.325 & 0.418 & 0.637 & 0.78 & 0.51 \\
RS & 0.649 & 0.798 & 1.108 & 0.81 & 0.58 \\
LTS & 0.568 & 0.678 & 0.896 & 0.84 & 0.63 \\
RTS & 0.669 & 0.896 & 1.276 & 0.75 & 0.52 \\
\hline \hline
\end{tabular}

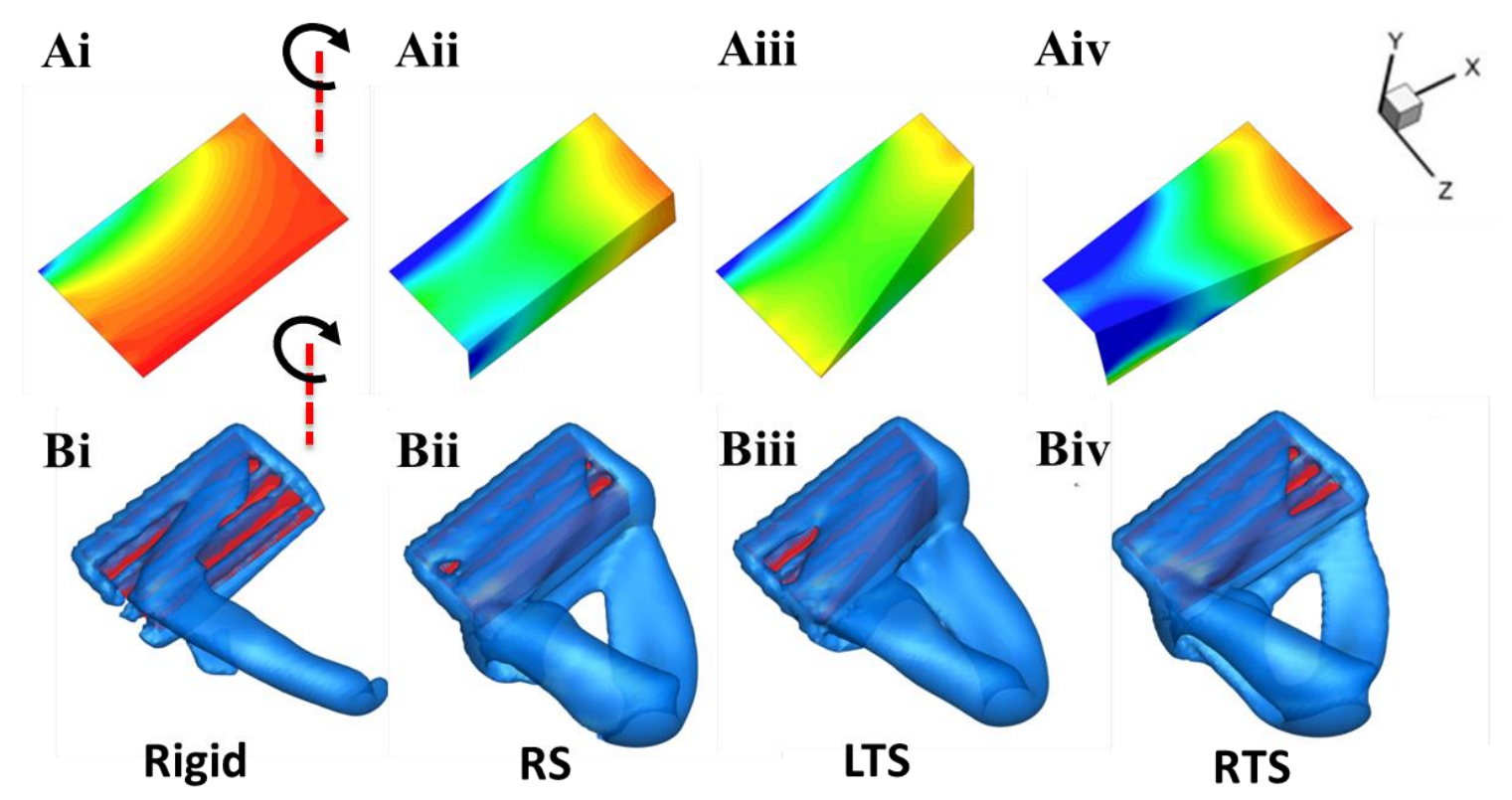

Figure 31: Comparison of surface pressure distribution on the suction side of the plate (A) and iso-surface vortex structure (B) for different trailing-edge shape at the middle of down-stroke 


\section{Chapter 5: Summary and Conclusions}

\subsection{Accomplishments}

A parametric study has been conducted to investigate the aerodynamic performance of deformed plates in hovering motion. A model of 2D flapping plate with dynamic trailing edge deviation angle is built. Two adjustable parameters trailing-edge deflection amplitude $\left(\beta_{T}\right)$ and phase shift between leading-edge and trailing-edge rotation $(\varphi)$ are used to control the trailing edge flap.

The results show that the effect of trailing-edge flap was amplified as the flow becoming more unsteady in terms of higher reduced frequency $(k)$. For a selected stroke amplitude $\left(A_{0} / c=3\right)$, flapping plate can increase the cycle-averaged lift productive in a relatively efficient way by considering higher lift production and lower power consumption. All pairs of trailing-edge deflection amplitude angle $\left(\beta_{T}=\left[10^{\circ}, 80^{\circ}\right]\right)$ and phase shift angle $\left(\varphi=\left[-80^{\circ}, 80^{\circ}\right]\right)$, at stroke amplitude fixed at $A_{0} / c=3$, were evaluated. From the aerodynamic performance contours, it is observed that the optimal lift production and optimal aerodynamic efficiency can be achieved by tailoring the trailingedge deflection angle and phase shift. However, this performance enhancement comes at a cost. It is always a compromise between high lift and high efficiency. In general, drag force and power consumption are more sensitive for the changing of deflection amplitude $\left(\beta_{T}\right)$. On the other hand, lift generation is more sensitive to the change of phase shift $(\varphi)$. 
The highest lift production and aerodynamic efficiency can be achieved by using the combination of $\beta_{T}=50^{\circ}, \varphi=+60^{\circ}$ and $\beta_{T}=10^{\circ}, \varphi=-80^{\circ}$, respectively.

Further analysis based on the flow fields showed that the dynamic motion of trailing-edge flap feeds extra circulation to the trailing-edge vortex which induced a stronger pair leading-edge vortex. As a result, the vortex structure on the suction side is pulled down closer to the plate and this leads to a larger range of low pressure field. Thus, the lift production is enhanced resulting from the increasing pressure difference between the upper and lower plate surface. The mean velocity in vertical direction also present a much stronger high-intensity jet for the plate with optional trailing-edge flap configuration comparing to the rigid flapping plate case.

Unsteady aerodynamic performance of deformed flapping plated has also been considered for three-dimensional flows. Three-dimensional effects such as aspect-ratio and geometry of trailing-edge flap have been studied. The results show that the larger aspect-ratio case owns higher aerodynamic efficiency in terms of lift-to-power ratio. Through applying different trailing-edge shape, the surface pressure distribution on the suction side will be changed and result in a vortex structure modulation. 


\subsection{Future Work}

The results from our analysis show that trailing-edge flap offers a viable alternate way for lift enhancement in hovering. In addition, the results from two-dimensional and three-dimensional parametric studies show that the significant improvement can be obtained by tailoring the trailing-edge deflection angle and phase shift. The following recommendations are mad for future work.

1. In order to investigate the effect of trailing-edge flap on the aerodynamic performance and flow modulation, the kinematics of flap was controlled by a prescribed sinusoidal function in present work. The further goal is to develop a closed-loop control method which can adjust the kinematics of trailing-edge flap instantaneously.

2. In addition, the current analysis shows that the structure of the leading-edge vortex (LEV) is affected significantly by changes in trailing-edge flap kinematics, such as amplitude and phase shift. In real birds or insects flapping flight, deformed wing present more complex deformation in both chord-wise and spanwise. So, more in-depth model could be developed to allow the plate to deform with the flow rather than the prescribed deflection.

3. Moreover, the focus of current was to use the idealized hovering motion to study the aerodynamic function of trailing-edge flap. This also can be adapted to further study other useful maneuver in flapping flight, such as forward flight, take-off, and turning maneuvers.

4. One of the requirements for a viable MAV is the ability to withstand sudden perturbations in the wind speed. Atmospheric turbulence dictated by terrain and 
weather conditions can potentially introduce roll, pitch and yaw disturbances. The MAV is expected to fly within $100 \mathrm{~m}$ from the ground where wakes shed by ground objects are also anticipated to play a significant role. Hence, analyzing influence of dynamic trailing-edge flap by changing the free-stream condition using an impulse function with varying frequency and amplitude is necessary to get a comprehensive understanding of the effect of wind gusts on lift and thrust production. 


\section{Bibliography}

[1] J.H.a.H. Mcmasters, M. L., Low Speed Single Element Airfoil Synthesis, Tec. Soaring 2 (1980).

[2] B.H. Carmichael, Low Reynolds Number Airfoil Survey, NASA Contractor Report (1981).

[3] A.a.M. Pelletier, T. J., Low Reynolds Number Aerodynamics of Low-Aspect-Ratio, Journal of Aircraft (2005) 825-832.

[4] S.K. Chimakurthi, J. Tang, R. Palacios, C.E.S. Cesnik, W. Shyy, Computational Aeroelasticity Framework for Analyzing Flapping Wing Micro Air Vehicles, AIAA Journal 47 (2009) 1865-1878.

[5] M. Hamamoto, Y. Ohta, K. Hara, T. Hisada, Application of fluid-structure interaction analysis to flapping flight of insects with deformable wings, Advanced Robotics 21 (2007) 1-21.

[6] B. Yin, H.X. Luo, Effect of wing inertia on hovering performance of flexible flapping wings, Physics of Fluids 22 (2010).

[7] S.M. Walker, A.L.R. Thomas, G.K. Taylor, Deformable wing kinematics in the desert locust: how and why do camber, twist and topography vary through the stroke?, Journal of the Royal Society Interface 6 (2009) 735-747.

[8] C. Koehler, Z.X. Liang, Z. Gaston, H. Wan, H.B. Dong, 3D reconstruction and analysis of wing deformation in free-flying dragonflies, Journal of Experimental Biology 215 (2012) 3018-3027.

[9] D.E. Alexander, Nature's Flyers, Johns Hopkins University Press, Baltimore, 2002. 
[10] F.M. Najjar, R. Mittal, Simulations of Complex Flows and Fluid-Structure Interaction Problems on Fixed Cartesian Grids, 4th ASME- JSME Joint Fluids Engineering Conference (2003).

[11] M. Bozkurttas, H. Dong, R. Mittal, F. Najjar, Towards Numerical Simulation of Flapping Foil on Fixed Cartesian grids, AIAA, RenoNV, 2005-0081.

[12] A.J. Chorin, Bull. Amer. Math. Soc. (1967) 73, 928-931.

[13] J.M. Stockie, B.R. Wetton, Analysis of stiffness in the immersed boundary method and implications for time-stepping schemes, 1998, pp. 41.

[14] R. Mittal, G. Iaccarino, Immersed boundary methods, Annual Review of Fluid Mechanics 37 (2005) 239-261.

[15] H. Dong, M. Bozkurttas, R. Mittal, P. Madden, G.V. Lauder, Computational modelling and analysis of the hydrodynamics of a highly deformable fish pectoral fin, Journal of Fluid Mechanics 645 (2010) 345-373.

[16] K. Taira, T. Colonius, Three-dimensional flows around low-aspect-ratio flat-plate wings at low Reynolds numbers, Journal of Fluid Mechanics 623 (2009) 187-207.

[17] B.C. Yun Liu, and Xinyan Deng, An experimental Study of Dynamic Trailing Edge Deflections on a Two Dimensional Translating Wing, AIAA, 2013, pp. 24-27.

[18] B.C. Yun Liu, Xinyan Deng, An application of smoke-wire visualization on a hovering insect wing, Journal of Visualization 16 (2013) 185-187.

[19] F.a.J. Manar, Anya R., The Eect of Tip Clearance on Low Reynolds Number Rotating Wings, AIAA Aerospace Sciences Meeting, National Harbor, Maryland, January, 2014. 
[20] C.W. J. Eldredge, and M. Ol, A computational study of a canonical pitch-up, pitchdown, wing maneuver, AIAA Paper No. 2009-3687 (2009).

[21] Z.J. Wang, The role of drag in insect hovering, J Exp Biol 207 (2004) 4147-4155.

[22] J. Leishman, Principles of Helicopter Aerodynamics, University of Cambridge, Cambridge, 2000.

[23] Z.J. Wang, J.M. Birch, M.H. Dickinson, Unsteady forces and flows in low Reynolds number hovering flight: two-dimensional computations vs robotic wing experiments, Journal of Experimental Biology 207 (2004) 449-460.

[24] T.J. Mueller, Fixed and flapping wing aerodynamics for micro air vehicle applications, American Institute of Aeronautics and Astronautices, Inc, Reston, Virginia, 2001.

[25] H. Dong, Z. Liang, M. Harff, Optimal Settings of Aerodynamic Performance Parameters in Hovering Flight, International Journal of Micro Air Vehicle 1 (2009) $173-181$.

[26] S. Heathcote, Wang, Z., and Gursul I., Effect of Spanwise Flexibility on Flapping Wing Propulsion, 36th AIAA Fluid Dynamics Conference (2006).

[27] S. Heathcote, I. Gursul, Flexible flapping airfoil propulsion at low Reynolds numbers, AIAA Journal 45 (2007) 1066-1079.

[28] M.H. Dickinson, F.O. Lehmann, S.P. Sane, Wing rotation and the aerodynamic basis of insect flight, Science 284 (1999) 1954-1960.

[29] M. Dickinson, Insect flight, Curr Biol 16 (2006) R309-314.

[30] M. Dickinson, Solving the mystery of insect flight, Sci Am 284 (2001) 48-57. 
[31] J.M. Anderson, K. Streitlien, D.S. Barrett, M.S. Triantafyllou, Oscillating foils of high propulsive efficiency, Journal of Fluid Mechanics 360 (1998) 41-72.

[32] G.C. Lewin, H. Haj-Hariri, Modelling thrust generation of a two-dimensional heaving airfoil in a viscous flow, 492 (2003) 339-362. 